\title{
Epidemic Spread and Variation of Peak Times in Connected Regions due to Travel-Related Infections-Dynamics of an Antigravity-Type Delay Differential Model $^{*}$
}

\author{
Diána H. Knipl ${ }^{\dagger}$, Gergely Röst ${ }^{\ddagger}$, and Jianhong $\mathrm{Wu}^{\S}$
}

\begin{abstract}
National boundaries have never prevented infectious diseases from reaching distant territories; however, the speed at which an infectious agent can spread around the world via the global airline transportation network has significantly increased during recent decades. We introduce an SEAIRbased, antigravity model to investigate the spread of an infectious disease in two regions which are connected by transportation. As a submodel, an age-structured system is constructed to incorporate the possibility of disease transmission during travel, where age is the time elapsed since the start of the travel. The model is equivalent to a large system of differential equations with dynamically defined delayed feedback. After describing fundamental but biologically relevant properties of the system, we detail the calculation of the basic reproduction number and obtain disease transmission dynamics results in terms of $\mathcal{R}_{0}$. We parametrize our model for influenza and use real demographic and air travel data for the numerical simulations. To understand the role of the different characteristics of the regions in the propagation of the disease, three distinct origin-destination pairs are considered. The model is also fitted to the first wave of the influenza A(H1N1) 2009 pandemic in Mexico and Canada. Our results highlight the importance of including travel time and disease dynamics during travel in the model: the invasion of disease-free regions is highly expedited by elevated transmission potential during transportation.
\end{abstract}

Key words. differential equations, transportation model, epidemic spread, influenza modeling

AMS subject classifications. Primary, 34K05; Secondary, 92D30

DOI. $10.1137 / 130914127$

1. Introduction. The global network of human transportation has played a paramount role in the spatial spread of infectious diseases. The high connectedness of distant territories by air travel makes it possible for a disease to invade regions far away from the source faster than ever. Some infectious diseases, such as tuberculosis, measles, and seasonal influenza, have been known to be transmissible during commercial flights. The importance of the global

\footnotetext{
* Received by the editors March 22, 2013; accepted for publication (in revised form) by J. Sneyd July 17, 2013; published electronically DATE. The work of the first and second authors was partly supported by the European Union and the European Social Fund through project FuturICT.hu (grant TÁMOP-4.2.2.C-11/1/KONV-2012-0013). The project was also subsidized by the European Union and co-financed by the European Social Fund.

http://www.siam.org/journals/siads/x-x/91412.html

${ }^{\dagger}$ MTA-SZTE Analysis and Stochastics Research Group, Hungarian Academy of Sciences, Hungary (knipl@math. u-szeged.hu). This author's work was partially supported by the TÁMOP 4.2.4. A/2-11-1-2012-0001 "National Excellence Program - Elaborating and operating an inland student and researcher personal support system convergence program."

¥Bolyai Institute, University of Szeged, Hungary (rost@math.u-szeged.hu). This author's work was partially supported by the European Research Council StG Nr. 259559.

${ }^{\S}$ Centre for Disease Modelling, Laboratory for Industrial and Applied Mathematics, York University, Toronto, ON, Canada (wujh@mathstat.yorku.ca). This author's work was partially supported by Natural Sciences and Engineering Research Council of Canada and by the Canada Research Chair Program.
} 
air travel network was highlighted in the 2002-2003 SARS outbreak (WHO [38]) and clearly contributed to the global spread of the 2009 pandemic influenza A(H1N1) (Khan et al. [15]). Therefore, mathematically describing the spread of infectious diseases on the global human transportation network is of critical public health importance.

There are a few well-known studies which constructed and analyzed various meta-population models for disease spread in connecting regions (see Arino [2], Arino and van den Driessche [3], Ruan, Wang, and Levin [25], Wang and Zhao [37], and the references therein). These studies focus mainly on the impact of spatial dispersal of infected individuals from one region to another, and do not consider transportation as a platform of disease dynamics. However, during long-distance travel, such as intercontinental flights, a single infected individual may infect several other passengers (Wagner, Coburn, and Blower [36], European Center for Disease Prevention an Control [12]), thus potentially inducing multiple generating infections in the destination region. It is therefore desirable to properly describe the spread of the disease via long-distance travel, in a way that incorporates into the models the transmission dynamics during the transition and travel.

Cui, Takeuchi, and Saito [8] and Takeuchi, Liu, and Cui [31] modeled the possibility that individuals may contract a disease while traveling by a system of ordinary differential equations based on the standard SIS (susceptible-infected-susceptible) epidemic model. They discovered that the disease can persist in regions connected by human transportation even if the infection died out in all regions in the absence of travel. Liu, Wu, and Zhou [18] noted that the previously proposed models $[8,19,31]$ implicitly used the assumption that the transportation between regions occur instantaneously. For some diseases of major public health concern, such as SARS and influenza, the progress of the disease is so fast that even a short delay (a fraction of a day) can be significant. Based on such considerations, Liu, Wu, and Zhou [18] introduced the time needed to complete the travel into the SIS-type epidemic model, and also the possible infections during this time. Nakata [22] described the global dynamics of this system for two identical regions in terms of the basic reproduction number. The model was later generalized by Nakata and Röst [23] to the case of $n$ regions with different characteristics and arbitrary travel networks. An SIR (susceptible-infected-recovered)-based model with a general incidence term was analyzed in Knipl and Röst [17] to describe the spread of infection in multiple regions with travel considered.

The purpose of this work is to formulate a model to properly describe the temporal evolution of an epidemic in regions connected by long-distance travel, such as intercontinental flights. The European Centre for Disease Prevention and Control (ECDC) developed a risk assessment guideline [12] for infectious diseases transmitted on aircrafts, like influenza. Existing studies confirmed that on-board transmission was possible in flights even with a duration of less than eight hours. For most diseases that pose a threat of a global pandemic, an SIS-type model is not adequate. For this reason, here we use the SEAIR model as a basic epidemic model building block in the regions and also during travel. The SIS model can be reduced to a logistic equation and then can be solved analytically. This property was heavily used in the analysis done in $[18,22,23]$. However, the lack of closed form solution causes substantial technical difficulties in the analysis of SEAIR-type models, as will be shown in this paper.

More significantly the aforementioned existing models did not distinguish local residents from temporary visitors in the model setup. In reality, the large part of travels are return 
trips, and not only the number of visitors, but also the average time that visitors spend in the other region may significantly affect the speed of spatial spread of the disease. If visitors spend more time in a region which is a hotspot of the disease, they will more likely carry the disease back to their region of origin. In addition, visitors and local residents may have very different contact rates and mixing patterns, for example if the visitors are typically on holiday and stay in selected resorts and hotels. Hence in our model we use different compartments for residents and visitors to capture this phenomenon.

Many multiregional epidemic models, specially the gravity-type models, are based on the assumption that the speed of the spread of epidemics between regions is inversely proportional to the distance between those regions (see, for example, Tuite et al. [32] for the recent cholera outbreak in Haiti). However, in case of air travel, the travel behavior is different and can be just the opposite. First, the number of travelers does not depend directly on the distance between regions, but is determined by other more important factors, such as business and cultural relations or touristic attractivity. Second, the transmission rate of an infectious disease can be much higher than usual when a large number of passengers are sharing the same cabin, and the longer the flight (which means the larger distance is between regions), the greater the number of infections that can be expected (Wagner, Coburn, and Blower [36]). Hence the air travel model we are proposing here is in principle "antigravity."

The paper is organized as follows. In the next section we formulate an age-structured model (where age means time since the start of travel), which leads to a nonlinear system of functional differential equations. In section 3 we determine some fundamental properties of the model. Section 4 is concerned with the computation of local and global reproduction numbers. We parametrize our model for influenza in section 5, and then in section 6 we introduce three prototype origin-destination pairs (Canada-Mexico, Canada-China, CanadaUK) and run simulations using real air traffic and tourism data. In the last section we discuss our findings.

2. Model description. We formulate a dynamical model describing the spread of an infectious disease within and between two regions, and also during travel from one region to the other. We divide the entire populations of the two regions into the disjoint classes $S_{j}^{m}$, $E_{j}^{m}, A_{j}^{m}, I_{j}^{m}, R_{j}^{m}, j \in\{1,2\}, m \in\{r, v\}$, where the letters $S, E, A, I$, and $R$ represent the compartments of susceptible, exposed, asymptomatic infected, symptomatic infected, and recovered individuals, respectively. Lower index $j \in\{1,2\}$ specifies the current region, upper index $m \in\{r, v\}$ denotes the residential status of the individual in the current region (resident versus visitor). For instance, $S_{1}^{v}$ is the compartment of individuals who are susceptible to the disease and staying in region 1 as a visitor (hence, they originally belong to region 2); members of $A_{2}^{r}$ are those who are asymptomatic infected residents in region 2.

Let $S_{j}^{m}(t), E_{j}^{m}(t), A_{j}^{m}(t), I_{j}^{m}(t), R_{j}^{m}(t), j \in\{1,2\}, m \in\{r, v\}$, be the number of individuals belonging to $S_{j}^{m}, E_{j}^{m}, A_{j}^{m}, I_{j}^{m}, R_{j}^{m}$, respectively, at time $t$. The transmission rate between an infected individual with residential status $m$ and a susceptible individual with residential status $n$ in region $j(j \in\{1,2\}, m, n \in\{r, v\})$ is denoted by $\beta_{j}^{m, n}$. Let $F_{j}^{r}$ denote the force of infection of residents, and $F_{j}^{v}$ the force of infection of visitors in region $j$. Model parameter $\mu_{E}$ denotes the inverse of the incubation period, $\mu_{A}$ and $\mu_{I}$ are the recovery rates of asymptomatic and symptomatic infected individuals. Let $\rho$ be the reduction factor of infectiousness 
of asymptomatic infected individuals (we assume they are capable of transmitting the disease, but generally with a lower rate than symptomatic infected individuals). Let $p$ denote the probability that an infected individual develops symptoms, and let $\delta$ denote disease-induced mortality rate. We assume constant recruitment terms $\Lambda_{j}$, while $d_{j}^{r}$ and $d_{j}^{v}$ denote natural mortality rates of residents and visitors in region $j$. We denote the travel rate of residents between region $j$ and region $k$ by $\alpha_{j}$, and the rate at which visitors of region $j$ travel back to region $k$ by $\gamma_{j}$; thus $1 / \gamma_{j}$ is the average time visitors spend in region $j$. For the total population of residents, visitors, and all individuals currently being in region $j$ at time $t$, we use the notation

$$
\begin{aligned}
& N_{j}^{r}(t)=S_{j}^{r}(t)+E_{j}^{r}(t)+A_{j}^{r}(t)+I_{j}^{r}(t)+R_{j}^{r}(t), \\
& N_{j}^{v}(t)=S_{j}^{v}(t)+E_{j}^{v}(t)+A_{j}^{v}(t)+I_{j}^{v}(t)+R_{j}^{v}(t), \\
& N_{j}(t)=N_{j}^{r}(t)+N_{j}^{v}(t) .
\end{aligned}
$$

We divide the population during travel into the classes $s_{j, k}^{m}, e_{j, k}^{m}, a_{j, k}^{m}, i_{j, k}^{m}, r_{j, k}^{m}$. Letters $s$, $e, a, i, r$ denote susceptible, exposed, asymptomatic infected, symptomatically infected, and recovered travelers, respectively. Lower indices $j, k \in\{1,2\}, j \neq k$, indicate that individuals are traveling from region $j$ to region $k$. Upper index $m \in\{r, v\}$ determines individuals' residential status in the region they have just left: for instance, an individual who is now in $r_{1,2}^{v}$ is recovered, traveling from region 1 to region 2 , and was a visitor in region 1, which means that the individual originally belongs to region 2 .

Let $\tau>0$ denote the average time required to complete a one-way trip. To describe the disease dynamics during travel, we define $s_{j, k}^{m}\left(\theta, t_{*}\right), e_{j, k}^{m}\left(\theta, t_{*}\right), a_{j, k}^{m}\left(\theta, t_{*}\right), i_{j, k}^{m}\left(\theta, t_{*}\right), r_{j, k}^{m}\left(\theta, t_{*}\right)$, $j, k \in\{1,2\}, j \neq k, m \in\{r, v\}$, as the density of individuals who started travel at time $t^{*}$ and belong to classes $s_{j, k}^{m}, e_{j, k}^{m}, a_{j, k}^{m}, i_{j, k}^{m}, r_{j, k}^{m}$ with respect to $\theta$, where $\theta \in[0, \tau]$ denotes the time elapsed since the beginning of the travel. Let

$$
n_{j, k}^{m}\left(\theta, t_{*}\right)=s_{j, k}^{m}\left(\theta, t_{*}\right)+e_{j, k}^{m}\left(\theta, t_{*}\right)+a_{j, k}^{m}\left(\theta, t_{*}\right)+i_{j, k}^{m}\left(\theta, t_{*}\right)+r_{j, k}^{m}\left(\theta, t_{*}\right),
$$

where $j, k \in\{1,2\}, j \neq k, m \in\{r, v\}$, and let

$$
n_{j, k}\left(\theta, t_{*}\right)=n_{j, k}^{r}\left(\theta, t_{*}\right)+n_{j, k}^{v}\left(\theta, t_{*}\right) .
$$

Thus, $\int_{\theta_{2}}^{\theta_{1}} n_{j, k}(\theta, t-\theta) d \theta$ is the number of individuals who left region $j$ in the time interval $\left[t-\theta_{1}, t-\theta_{2}\right]$, where $\tau \geq \theta_{1} \geq \theta_{2} \geq 0$. In particular, for $\theta_{1}=\tau$ and $\theta_{2}=0$, this gives the total number of individuals who are in the travel transition from region $j$ to region $k$ at time $t$. We assume that infected individuals do not die during travel; hence $n_{j, k}\left(\theta, t_{*}\right)=n_{j, k}\left(0, t_{*}\right)$ for all $\theta \in[0, \tau]$. During the course of travel, infected individuals can transmit the disease at the rate $\beta^{T}$. We use the notation $\mu_{E}^{T}, \mu_{A}^{T}, \mu_{I}^{T}$ for the inverse of the incubation period and the recovery rates of asymptomatic and symptomatic infected individuals during travel. Let $F_{j, k}^{T}$ denote the force of infection during travel from region $j$ to region $k$. Then $s_{j, k}^{m}(\tau, t-\tau)$, $e_{j, k}^{m}(\tau, t-\tau), a_{j, k}^{m}(\tau, t-\tau), i_{j, k}^{m}(\tau, t-\tau), r_{j, k}^{m}(\tau, t-\tau)$ gives the inflow of individuals arriving from region $j$ to compartments $S_{k}^{n}, E_{k}^{n}, A_{k}^{n}, I_{k}^{n}, R_{k}^{n}, j, k \in\{1,2\}, j \neq k, m, n \in\{r, v\}, m \neq n$, respectively, at time $t$.

All variables and model parameters are listed in Tables 1 and 2. The flow chart of the model is depicted in Figure 1. Based on the assumptions formulated above, we obtain the following system of differential equations for disease transmission in the two regions: 
Table 1

Model variables $(j, k \in\{1,2\}, j \neq k)$. In the figure, "density" means the density with respect to the age since the start of travel.

\begin{tabular}{|c|c|}
\hline \multicolumn{2}{|r|}{ Variables } \\
\hline$F_{j}^{r}$ & Force of infection of residents in region $j$ \\
\hline$F_{j}^{v}$ & Force of infection of visitors in region $j$ \\
\hline$F_{j, k}^{T}$ & Force of infection during travel from region $j$ to region $k$ \\
\hline$S_{j}^{r}, E_{j}^{r}, A_{j}^{r}, I_{j}^{r}, R_{j}^{r}$ & $\begin{array}{l}\text { Susceptible, exposed, asymptomatic, symptomatic } \\
\text { infected, or recovered residents in region } j\end{array}$ \\
\hline$S_{j}^{v}, E_{j}^{v}, A_{j}^{v}, I_{j}^{v}, R_{j}^{v}$ & $\begin{array}{l}\text { Susceptible, exposed, asymptomatic, symptomatic } \\
\text { or infected, recovered visitors in region } j\end{array}$ \\
\hline$N_{j}^{r}, N_{j}^{v}, N_{j}$ & $\begin{array}{l}\text { Total population size of residents, visitors, and } \\
\text { all individuals in region } j\end{array}$ \\
\hline$s_{j, k}^{r}, e_{j, k}^{r}, a_{j, k}^{r}, i_{j, k}^{r}, r_{j, k}^{r}$ & $\begin{array}{l}\text { Density of susceptible, exposed, asymptomatic, } \\
\text { symptomatic infected, or recovered individuals } \\
\text { during the travel from } j \text { to } k \text { (traveling to visit } k \text { ) }\end{array}$ \\
\hline$s_{j, k}^{v}, e_{j, k}^{v}, a_{j, k}^{v}, i_{j, k}^{v}, r_{j, k}^{v}$ & $\begin{array}{l}\text { Density of susceptible, exposed, asymptomatic, } \\
\text { symptomatic infected, or recovered individuals during } \\
\text { the travel from } j \text { to } k \text { (returning to } k \text { from visiting } j \text { ) }\end{array}$ \\
\hline$n_{j, k}^{r}, n_{j, k}^{v}, n_{j, k}$ & $\begin{array}{l}\text { Total density of residents, visitors, and } \\
\text { all individuals during the travel from } j \text { to } k\end{array}$ \\
\hline
\end{tabular}

Table 2

Key model parameters $(j, k \in\{1,2\}, j \neq k)$.

\begin{tabular}{|c|c|}
\hline \multicolumn{2}{|r|}{ Key model parameters } \\
\hline$\Lambda_{j}$ & Recruitment rate in region $j$ \\
\hline $\begin{array}{l}d_{j}^{r}, d_{j}^{v} \\
\quad \delta\end{array}$ & $\begin{array}{l}\text { Natural death rate of residents and visitors of region } j \\
\text { disease-induced death rate }\end{array}$ \\
\hline$\beta_{j}^{m, n}$ & $\begin{array}{l}\text { Transmission rate between an infected individual } \\
\text { with residential status } m \text { and a susceptible individual } \\
\text { with residential status } n \text { in region } j(m, n \in\{r, v\})\end{array}$ \\
\hline$\beta^{T}$ & Transmission rate during the travel \\
\hline$\alpha_{j}$ & Traveling rate of residents of region $j$ to region $k$ \\
\hline$\gamma_{j}$ & Inverse of duration of visitors' stay in region $j$ \\
\hline$\tau$ & Duration of travel between the regions \\
\hline$p$ & Probability of developing symptoms \\
\hline$\rho$ & Reduction of infectiousness of asymptotic infecteds \\
\hline$\mu_{E}, \mu_{E}^{T}$ & $\begin{array}{l}\text { Reciprocal of the length of the incubation period } \\
\text { in the regions and during the travel }\end{array}$ \\
\hline$\mu_{A}, \mu_{A}^{T}$ & $\begin{array}{l}\text { Recovery rate of asymptomatic infecteds } \\
\text { in the regions and during the travel }\end{array}$ \\
\hline$\mu_{I}, \mu_{I}^{T}$ & $\begin{array}{l}\text { Recovery rate of symptomatic infecteds } \\
\text { in the regions and during the travel }\end{array}$ \\
\hline
\end{tabular}




\section{Region $1 \quad$ Region 2}

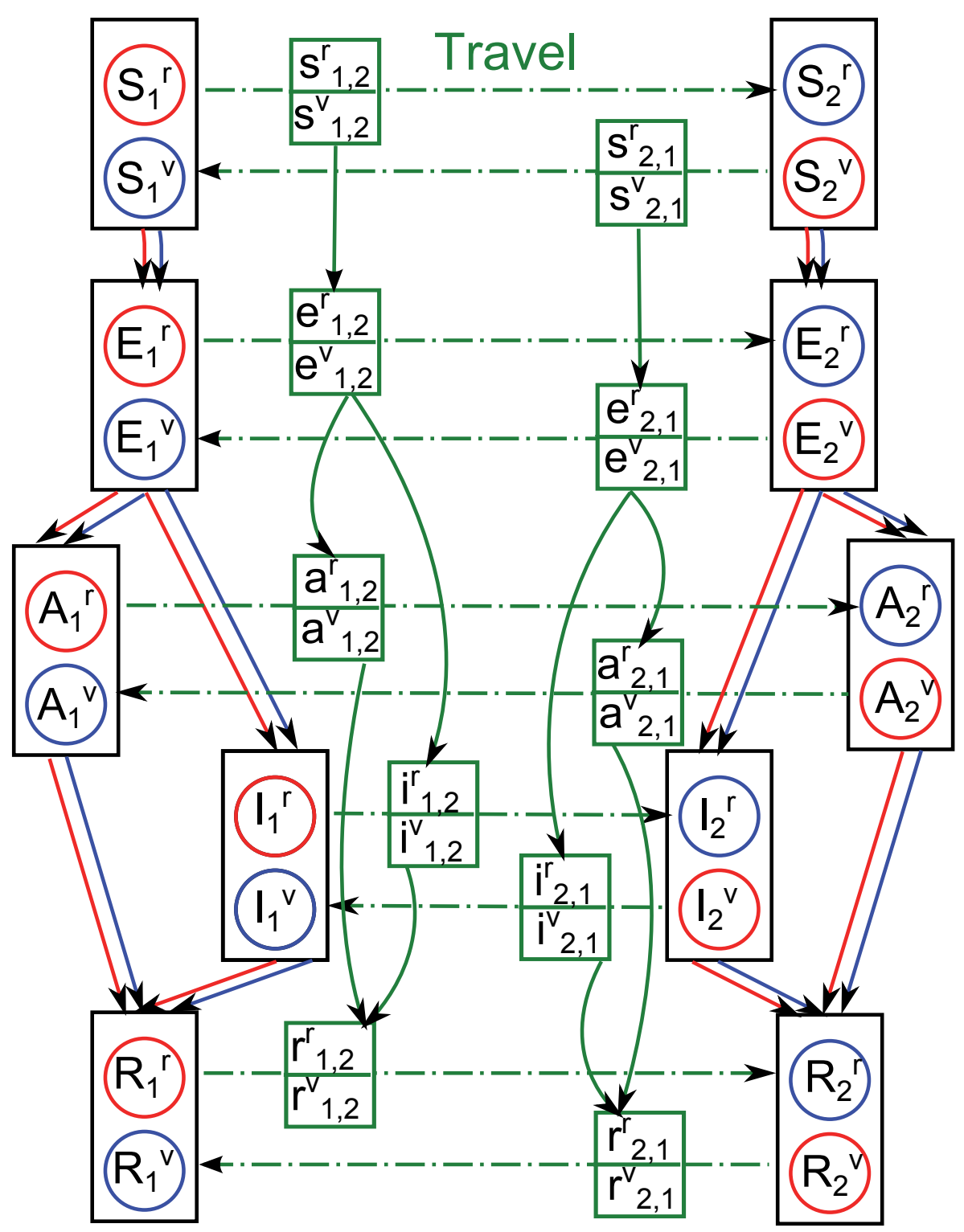

Figure 1. Color-coded flow chart of disease transmission and travel dynamics. The disease transmission in the two regions is shown in two different columns; the disease progresses vertically from top to bottom. Classes having the same origins are marked by the same colors. Red corresponds to the classes originating from region 1; blue represents classes of region 2. Arrows colored with the same colors indicate how the disease progresses. Green dashed-dotted arrows represent individuals that are traveling. Green solid arrows show the dynamics of the pandemic during the course of the travel. The description of the variables can be found in Table 1. 
$(L)$

$$
\left\{\begin{array}{l}
\dot{S}_{j}^{r}(t)=\Lambda_{j}-S_{j}^{r}(t) F_{j}^{r}(t)-\left(d_{j}^{r}+\alpha_{j}\right) S_{j}^{r}(t)+s_{k, j}^{v}(\tau, t-\tau), \\
\dot{E}_{j}^{r}(t)=S_{j}^{r}(t) F_{j}^{r}(t)-\left(d_{j}^{r}+\mu_{E}+\alpha_{j}\right) E_{j}^{r}(t)+e_{k, j}^{v}(\tau, t-\tau), \\
\dot{A}_{j}^{r}(t)=(1-p) \mu_{E} E_{j}-\left(d_{j}^{r}+\alpha_{j}+\mu_{A}\right) A_{j}^{r}(t)+a_{k, j}^{v}(\tau, t-\tau), \\
\dot{I}_{j}^{r}(t)=p \mu_{E} E_{j}-\left(d_{j}^{r}+\alpha_{j}+\delta+\mu_{I}\right) I_{j}^{r}(t)+i_{k, j}^{v}(\tau, t-\tau), \\
\dot{R}_{j}^{r}(t)=\mu_{I} I_{j}^{r}(t)+\mu_{A} A_{j}^{r}(t)-\left(d_{j}^{r}+\alpha_{j}\right) R_{j}^{r}(t)+r_{k, j}^{v}(\tau, t-\tau), \\
\dot{S}_{j}^{v}(t)=-S_{j}^{v}(t) F_{j}^{v}(t)-\left(d_{j}^{v}+\gamma_{j}\right) S_{j}^{v}(t)+s_{k, j}^{r}(\tau, t-\tau), \\
\dot{E}_{j}^{v}(t)=S_{j}^{v}(t) F_{j}^{v}(t)-\left(d_{j}^{v}+\mu_{E}+\gamma_{j}\right) E_{j}^{v}(t)+e_{k, j}^{r}(\tau, t-\tau), \\
\dot{A}_{j}^{v}(t)=(1-p) \mu_{E} E_{j}^{v}(t)-\left(d_{j}^{v}+\gamma_{j}+\mu_{A}\right) A_{j}^{v}(t)+a_{k, j}^{r}(\tau, t-\tau), \\
\dot{I}_{j}^{v}(t)=p \mu_{E} E_{j}^{v}(t)-\left(d_{j}^{v}+\gamma_{j}+\delta+\mu_{I}\right) I_{j}^{v}(t)+i_{k, j}^{r}(\tau, t-\tau), \\
\dot{R}_{j}^{v}(t)=\mu_{I} I_{j}^{v}(t)+\mu_{A} A_{j}^{v}(t)-\left(d_{j}^{v}+\gamma_{j}\right) R_{j}^{v}(t)+r_{k, j}^{r}(\tau, t-\tau),
\end{array}\right.
$$

where

$$
\begin{aligned}
& F_{j}^{r}(t)=\frac{1}{N_{j}(t)}\left(\beta_{j}^{r r}\left(I_{j}^{r}(t)+\rho A_{j}^{r}(t)\right)+\beta_{j}^{v r}\left(I_{j}^{v}(t)+\rho A_{j}^{v}(t)\right)\right), \\
& F_{j}^{v}(t)=\frac{1}{N_{j}(t)}\left(\beta_{j}^{r v}\left(I_{j}^{r}(t)+\rho A_{j}^{r}(t)\right)+\beta_{j}^{v v}\left(I_{j}^{v}(t)+\rho A_{j}^{v}(t)\right)\right) .
\end{aligned}
$$

For each given $t_{*}$, the following system $(T)$ describes the evolution of the densities during the travel initiated at time $t_{*}$ :

$$
\left\{\begin{array}{l}
\frac{d}{d \theta} s_{j, k}^{r}\left(\theta, t_{*}\right)=-s_{j, k}^{r}\left(\theta, t_{*}\right) F_{j, k}^{T}\left(\theta, t_{*}\right) \\
\frac{d}{d \theta} e_{j, k}^{r}\left(\theta, t_{*}\right)=s_{j, k}^{r}\left(\theta, t_{*}\right) F_{j, k}^{T}\left(\theta, t_{*}\right)-\mu_{E}^{T} e_{j, k}^{r}\left(\theta, t_{*}\right), \\
\frac{d}{d \theta} a_{j, k}^{r}\left(\theta, t_{*}\right)=(1-p) \mu_{E}^{T} e_{j, k}^{r}\left(\theta, t_{*}\right)-\mu_{A}^{T} a_{j, k}^{r}\left(\theta, t_{*}\right), \\
\frac{d}{d \theta} i_{j, k}^{r}\left(\theta, t_{*}\right)=p \mu_{E}^{T} e_{j, k}^{r}\left(\theta, t_{*}\right)-\mu_{I}^{T} i_{j, k}^{r}\left(\theta, t_{*}\right) \\
\frac{d}{d \theta} r_{j, k}^{r}\left(\theta, t_{*}\right)=\mu_{A}^{T} a_{j, k}^{r}\left(\theta, t_{*}\right)+\mu_{I}^{T} i_{j, k}^{r}\left(\theta, t_{*}\right) \\
\frac{d}{d \theta} s_{j, k}^{v}\left(\theta, t_{*}\right)=-s_{j, k}^{v}\left(\theta, t_{*}\right) F_{j, k}^{T}\left(\theta, t_{*}\right) \\
\frac{d}{d \theta} e_{j, k}^{v}\left(\theta, t_{*}\right)=s_{j, k}^{v}\left(\theta, t_{*}\right) F_{j, k}^{T}\left(\theta, t_{*}\right)-\mu_{E}^{T} e_{j, k}^{v}\left(\theta, t_{*}\right), \\
\frac{d}{d \theta} a_{j, k}^{v}\left(\theta, t_{*}\right)=(1-p) \mu_{E}^{T} e_{j, k}^{v}\left(\theta, t_{*}\right)-\mu_{A}^{T} a_{j, k}^{v}\left(\theta, t_{*}\right), \\
\frac{d}{d \theta} i_{j, k}^{v}\left(\theta, t_{*}\right)=p \mu_{E}^{T} e_{j, k}^{v}\left(\theta, t_{*}\right)-\mu_{I}^{T} i_{j, k}^{v}\left(\theta, t_{*}\right) \\
\frac{d}{d \theta} r_{j, k}^{v}\left(\theta, t_{*}\right)=\mu_{A}^{T} a_{j, k}^{v}\left(\theta, t_{*}\right)+\mu_{I}^{T} i_{j, k}^{v}\left(\theta, t_{*}\right)
\end{array}\right.
$$


where $j, k \in\{1,2\}, j \neq k$, and

$$
\begin{aligned}
F_{j, k}^{T}\left(\theta, t_{*}\right)= & \frac{\beta^{T}}{n_{j, k}\left(\theta, t_{*}\right)}\left(i_{j, k}^{r}\left(\theta, t_{*}\right)+i_{j, k}^{v}\left(\theta, t_{*}\right)+\rho\left(a_{j, k}^{r}\left(\theta, t_{*}\right)+a_{j, k}^{v}\left(\theta, t_{*}\right)\right)\right), \\
n_{j, k}\left(\theta, t_{*}\right)= & \alpha_{j}\left(S_{j}^{r}\left(t_{*}\right)+E_{j}^{r}\left(t_{*}\right)+A_{j}^{r}\left(t_{*}\right)+I_{j}^{r}\left(t_{*}\right)+R_{j}^{r}\left(t_{*}\right)\right) \\
& \quad+\gamma_{j}\left(S_{j}^{v}\left(t_{*}\right)+E_{j}^{v}\left(t_{*}\right)+A_{j}^{v}\left(t_{*}\right)+I_{j}^{v}\left(t_{*}\right)+R_{j}^{v}\left(t_{*}\right)\right) \\
= & \alpha_{j} N_{j}^{r}\left(t_{*}\right)+\gamma_{j} N_{j}^{v}\left(t_{*}\right) .
\end{aligned}
$$

For $\theta=0$, the densities are determined by the rates at which individuals start their travels from one region to the other at time $t_{*}$. Hence, the initial values for system $(T)$ at $\theta=0$ are given by

$$
\left\{\begin{array}{cc}
s_{j, k}^{r}\left(0, t_{*}\right)=\alpha_{j} S_{j}^{r}\left(t_{*}\right), & s_{j, k}^{v}\left(0, t_{*}\right)=\gamma_{j} S_{1}^{v}\left(t_{*}\right), \\
e_{j, k}^{r}\left(0, t_{*}\right)=\alpha_{j} E_{j}^{r}\left(t_{*}\right), & e_{j, k}^{v}\left(0, t_{*}\right)=\gamma_{j} E_{j}^{v}\left(t_{*}\right), \\
a_{j, k}^{r}\left(0, t_{*}\right)=\alpha_{j} A_{j}^{r}\left(t_{*}\right), & a_{j, k}^{v}\left(0, t_{*}\right)=\gamma_{j} A_{j}^{v}\left(t_{*}\right), \\
i_{j, k}^{r}\left(0, t_{*}\right)=\alpha_{j} I_{j}^{r}\left(t_{*}\right), & i_{j, k}^{v}\left(0, t_{*}\right)=\gamma_{j} I_{j}^{v}\left(t_{*}\right), \\
r_{j, k}^{r}\left(0, t_{*}\right)=\alpha_{j} R_{j}^{r}\left(t_{*}\right), & r_{j, k}^{v}\left(0, t_{*}\right)=\gamma_{j} R_{j}^{v}\left(t_{*}\right)
\end{array}\right.
$$

for $j, k \in\{1,2\}, j \neq k$.

Now we turn our attention to the terms $s_{j, k}^{m}(\tau, t-\tau), e_{j, k}^{m}(\tau, t-\tau), a_{j, k}^{m}(\tau, t-\tau), i_{j, k}^{m}(\tau, t-\tau)$, $r_{j, k}^{m}(\tau, t-\tau)$ in system $(L)$, which are the densities of individuals arriving to classes $S_{k}^{n}, E_{k}^{n}$, $A_{k}^{n}, I_{k}^{n}, R_{k}^{n}, j, k \in\{1,2\}, j \neq k, m, n \in\{r, v\}, m \neq n$, respectively, at time $t$, upon completing a one-way trip from region $j$. At time $t$, these terms are determined by the solution of system $(T)$ with initial values $(I V T)$ for $t_{*}=t-\tau$ at $\theta=\tau$ :

(i) individuals who enter region $k$ at time $t$ are those who left region $j$ at time $t-\tau$;

(ii) residents of region $j$ become visitors of region $k$ and vice versa $(m \neq n)$ upon completing a one-way trip;

(iii) an individual may move to a different compartment during travel; for example, a susceptible resident who travels from region $j$ may arrive as an infected visitor to region $k$ $(j, k \in\{1,2\}, j \neq k)$, as given by the dynamics of system $(T)$.

Next we specify initial values for system $(L)$ at $t=0$. Since travel takes $\tau$ units of time to complete, arrivals to region $j$ are determined by the state of region $k(j, k \in\{1,2\}, j \neq k)$ at $t-\tau$, via the solution of systems $(T)$ and $(I V T)$. Thus, we set up initial functions as follows:

$$
\left\{\begin{aligned}
S_{j}^{r}(u)=\varphi_{S, j}^{r}(u), & S_{j}^{v}(u)=\varphi_{S, j}^{v}(u), \\
E_{j}^{r}(u)=\varphi_{E, j}^{r}(u), & E_{j}^{v}(u)=\varphi_{E, j}^{v}(u) \\
A_{j}^{r}(u)=\varphi_{A, j}^{r}(u), & A_{j}^{v}(u)=\varphi_{A, j}^{v}(u) \\
I_{j}^{r}(u)=\varphi_{I, j}^{r}(u), & I_{j}^{v}(u)=\varphi_{I, j}^{v}(u) \\
R_{j}^{r}(u)=\varphi_{R, j}^{r}(u), & R_{j}^{v}(u)=\varphi_{R, j}^{v}(u)
\end{aligned}\right.
$$

where $u \in[-\tau, 0]$, and each $\varphi_{K, j}^{m}$ is a continuous function for $j \in\{1,2\}, m \in\{v, r\}, K \in$ $\{S, E, A, I, R\}$. 
Note that systems $(L)$ and $(T)$ are interconnected; in order to determine the dynamics of the model, simultaneous solution of both is required. Considering the fact that disease transmission is possible during travel, the solution of system $(T)$ at $(\tau, t-\tau)$ is required for all $t \geq 0$ to find the solution of $(L)$. However, in order to obtain the solution of $(T)$ at $(\tau, t-\tau)$, it is necessary to use the solution of $(L)$ at $t-\tau$, because $(T)$ takes the initial conditions from $(L)$. Hence, in order to describe the disease transmission in the regions, the solution of another differential equation system is required at each time $t$, which has initial values depending on the earlier state of the system on the regions. Thus $(L)$ is a delay differential system, where the delayed feedback is determined by a solution of a parallel system of ordinary differential equations. In previous papers with travel delay, such as $[18,22,23]$, the authors used an SIS-type system during travel, which was analytically solvable; thus it was possible to express the delayed feedback explicitly. Unlike the SIS model, the SEAIR model is not analytically solvable; therefore here we have to deal with a system of functional differential equations, where the delay term is given only implicitly via a solution of a nonlinear system of ordinary differential equations.

3. Basic properties of the model. In this section, we show that our model is equivalent to a system of nonlinear functional differential equations where the delay term is defined dynamically, via the solution of another system of differential equations. Then we also investigate some biologically relevant properties of the system. Set

$$
X_{j}^{r}=\left(\begin{array}{c}
S_{j}^{r} \\
E_{j}^{r} \\
A_{j}^{r} \\
I_{j}^{r} \\
R_{j}^{r}
\end{array}\right), \quad X_{j}^{v}=\left(\begin{array}{c}
S_{j}^{v} \\
E_{j}^{v} \\
A_{j}^{v} \\
I_{j}^{v} \\
R_{j}^{v}
\end{array}\right), \quad x_{j, k}^{r}=\left(\begin{array}{c}
s_{j, k}^{r} \\
e_{j, k}^{r} \\
a_{j, k}^{r} \\
i_{j, k}^{r} \\
r_{j, k}^{r}
\end{array}\right), \quad x_{j, k}^{v}=\left(\begin{array}{c}
s_{j, k}^{v} \\
e_{j, k}^{v} \\
a_{j, k}^{v} \\
i_{j, k}^{v} \\
r_{j, k}^{v}
\end{array}\right),
$$

where $j, k \in\{1,2\}, j \neq k$, and set

$$
X=\left(\begin{array}{c}
X_{1}^{r} \\
X_{1}^{v} \\
X_{2}^{r} \\
X_{2}^{v}
\end{array}\right), \quad x=\left(\begin{array}{l}
x_{2,1}^{v} \\
x_{2,1}^{r} \\
x_{1,2}^{v} \\
x_{1,2}^{r}
\end{array}\right)
$$

so that $X \in \mathbb{R}^{20}$ and $x \in \mathbb{R}^{20}$. For a given $t_{*}$ we define the system $\left(T^{*}\right)$ as

$$
\begin{aligned}
\frac{\partial}{\partial \theta} x\left(\theta, t_{*}\right) & =f\left(x\left(\theta, t_{*}\right)\right), \\
x\left(0, t_{*}\right) & =g\left(X\left(t_{*}\right)\right),
\end{aligned}
$$

where $t_{*}, \theta \in \mathbb{R}_{+}, f, g: \mathbb{R}^{20} \mathbb{R}^{20}$,

$$
g_{i}(y)= \begin{cases}\gamma_{2} y_{i} & \text { if } i=1, \ldots, 5 \\ \alpha_{2} y_{i} & \text { if } i=6, \ldots, 10 \\ \gamma_{1} y_{i} & \text { if } i=11, \ldots, 15 \\ \alpha_{1} y_{i} & \text { if } i=16, \ldots, 20\end{cases}
$$


and $f_{i}(x)$ equals the right-hand side of the equation for $x_{i}$ in system $(T)$. For instance,

$$
f_{7}(x)=\beta^{T} \frac{x_{6}}{\sum_{j=1}^{10} x_{j}}\left(x_{4}+x_{9}+\rho\left(x_{3}+x_{8}\right)\right)-\mu_{E}^{T} x_{7} .
$$

Define $G: \mathbb{R}^{20} \mathbb{R}^{20}$, where $G_{i}(X)$ is given by the right-hand side of the equation of $X_{i}$ in $(L)$ without the inflow from travel. For instance,

$$
G_{16}(X)=-\frac{X_{16}}{\sum_{j=11}^{20} X_{j}}\left(\beta_{2}^{r v}\left(X_{14}+\rho X_{13}\right)+\beta_{2}^{v v}\left(X_{19}+\rho X_{18}\right)\right)-\left(d_{2}^{v}+\gamma_{2}\right) X_{16} .
$$

Let $\hat{x}\left(\theta, t_{*} ; Y\right)$ denote the solution of the initial value problem $\left(T^{*}\right)$ for $t_{*}$ with initial value $\hat{x}\left(0, t_{*}\right)=g(Y)$, where $Y \in \mathbb{R}^{20}$, and let $H(Y):=\hat{x}(\tau, t-\tau ; Y), H: \mathbb{R}^{20} \mathbb{R}^{20}$. Then our system $(L)$ can be written in closed form as a system of functional differential equations,

$$
\dot{X}(t)=\mathcal{F}(X(t), X(t-\tau)),
$$

where $\mathcal{F}(X(t), X(t-\tau))=G(X(t))+H(X(t-\tau)), \mathcal{F}: \mathbb{R}^{20} \times \mathbb{R}^{20} \mathbb{R}^{20}$. Clearly $\left(T^{*}\right)$ is also a compact form of $(T)$. To study the dynamics of $\left(L^{*}\right)$, we define our phase space as the nonnegative cone $\mathcal{C}_{+}=\mathcal{C}\left([-\tau, 0], \mathbb{R}_{+}^{20}\right)$ of the Banach space of continuous functions from $[-\tau, 0]$ to $\mathbb{R}^{20}$, equipped with the supremum norm. For each $\Phi \in \mathcal{C}_{+}$, standard arguments guarantee that there exists a unique solution of system $\left(L^{*}\right)$ with initial values $X(u)=\Phi(u)$, $u \in[-\tau, 0]$ (see $[16,17])$. Using the notation of $(I V L)$, we have $\Phi=\left(\Phi_{1}^{r}, \Phi_{1}^{v}, \Phi_{2}^{r}, \Phi_{2}^{v}\right)^{T}$, where $\Phi_{j}^{r}=\left(\varphi_{S, j}^{r}, \varphi_{E, j}^{r}, \varphi_{A, j}^{r}, \varphi_{I, j}^{r}, \varphi_{R, j}^{r}\right)^{T}, \Phi_{j}^{v}=\left(\varphi_{S, j}^{v}, \varphi_{E, j}^{v}, \varphi_{A, j}^{v}, \varphi_{I, j}^{v}, \varphi_{R, j}^{v}\right)^{T}, j \in\{1,2\}$.

Proposition 1. For any $\Phi \in \mathcal{C}_{+}$, the solution of system $\left(L^{*}\right)$ is nonnegative.

Proof. It is straightforward to see that $\left(T^{*}\right)$ preserves nonnegativity; thus $H(Y) \geq 0$ if $Y \geq 0$. Since our function $\mathcal{F}(y, z): \mathbb{R}_{+}^{20} \times \mathbb{R}_{+}^{20} \mathbb{R}^{20}$ and $\mathcal{F}_{y}(y, z)$ are continuous on $\mathbb{R}^{20}$ and for every $i=1, \ldots 20$, for every $y, z \in \mathbb{R}_{+}^{20}, y_{i}=0$ implies $\mathcal{F}_{i}(y, z) \geq 0$, all the conditions of Theorem 3.4 in [27] hold. This implies that for nonnegative initial data the corresponding solution of system $\left(L^{*}\right)$ remains nonnegative.

We define the disease-free subspace $\mathcal{C}_{+}^{d f}$ as

$$
\mathcal{C}_{+}^{d f}=\left\{\Phi \mid \Phi=\left(\varphi_{S, j}^{r}, \hat{0}, \hat{0}, \hat{0}, \varphi_{R, j}^{r}, \varphi_{S, j}^{v}, \hat{0}, \hat{0}, \hat{0}, \varphi_{R, j}^{v}\right)^{T}\right\} \subset \mathcal{C}_{+},
$$

where $\hat{0}$ denotes the constant 0 function. If $\Phi \in \mathcal{C}_{+}^{d f}$, then

$$
E_{j}^{r}(t)=E_{j}^{v}(t)=A_{j}^{r}(t)=A_{j}^{v}(t)=I_{j}^{r}(t)=I_{j}^{v}(t) \equiv 0
$$

for all $t \geq 0$, and hence the disease-free subspace is positively invariant.

Proposition 2. In the disease-free subspace $\mathcal{C}_{+}^{d f}$ there exists a unique positive equilibrium of system $\left(L^{*}\right)$ which is globally asymptotically stable in $\mathcal{C}_{+}^{d f}$.

Proof. Using the definition of $N_{j}^{r}$ and $N_{j}^{v}$ (see Table 1) in section 2, for these variables we derive the following differential equation system:

$$
\begin{aligned}
& \dot{N}_{j}^{r}(t)=\Lambda_{j}-\left(d_{j}^{r}+\alpha_{j}\right) N_{j}^{r}(t)+\gamma_{k} N_{k}^{v}(t-\tau), \\
& \dot{N}_{j}^{v}(t)=-\left(d_{j}^{v}+\gamma_{j}\right) N_{j}^{v}(t)+\alpha_{k} N_{k}^{r}(t-\tau),
\end{aligned}
$$


where $j, k \in\{1,2\}, j \neq k$. One can find that the positive equilibrium $\left(\hat{N}_{1}^{r}, \hat{N}_{1}^{v}, \hat{N}_{2}^{r}, \hat{N}_{2}^{v}\right)$ is given by

$$
\left(\begin{array}{c}
\hat{N}_{1}^{r} \\
\hat{N}_{1}^{v} \\
\hat{N}_{2}^{r} \\
\hat{N}_{2}^{v}
\end{array}\right)=\left(\begin{array}{cccc}
d_{1}^{r}+\alpha_{1} & 0 & 0 & -\gamma_{2} \\
0 & d_{1}^{v}+\gamma_{1} & -\alpha_{2} & 0 \\
0 & -\gamma_{2} & d_{2}^{r}+\alpha_{2} & 0 \\
-\alpha_{2} & 0 & 0 & d_{2}^{v}+\gamma_{2}
\end{array}\right)^{-1}\left(\begin{array}{c}
\Lambda_{1} \\
0 \\
\Lambda_{2} \\
0
\end{array}\right)
$$

Set $M_{j}^{r}(t):=N_{j}^{r}(t)-\hat{N}_{j}^{r}, M_{j}^{v}(t):=N_{j}^{v}(t)-\hat{N}_{j}^{v}, j \in\{1,2\}$. We obtain the decoupled linear systems

$$
\begin{aligned}
& \dot{M}_{1}^{r}(t)=-\left(d_{1}^{r}+\alpha_{1}\right) M_{1}^{r}(t)+\gamma_{2} M_{2}^{v}(t-\tau), \\
& \dot{M}_{2}^{v}(t)=-\left(d_{2}^{v}+\gamma_{2}\right) M_{2}^{v}(t)+\alpha_{1} M_{1}^{r}(t-\tau),
\end{aligned}
$$

and

$$
\begin{aligned}
& \dot{M}_{2}^{r}(t)=-\left(d_{2}^{r}+\alpha_{2}\right) M_{2}^{r}(t)+\gamma_{1} M_{1}^{v}(t-\tau), \\
& \dot{M}_{1}^{v}(t)=-\left(d_{1}^{v}+\gamma_{1}\right) M_{1}^{v}(t)+\alpha_{2} M_{2}^{r}(t-\tau) .
\end{aligned}
$$

Since $d_{1}^{r}, d_{1}^{v}, d_{2}^{r}, d_{2}^{v}$ and $\alpha_{1}, \alpha_{2}, \gamma_{1}, \gamma_{2}$ are positive, and since $\left(d_{1}^{r}+\alpha_{1}\right)\left(d_{2}^{v}+\gamma_{2}\right)>\alpha_{1} \gamma_{2}$ and $\left(d_{2}^{r}+\alpha_{2}\right)\left(d_{1}^{v}+\gamma_{1}\right)>\alpha_{2} \gamma_{1}$, condition (16) in [30] holds. Thus, the zero solutions of systems (6) and (7) are asymptotically stable, which implies that the positive equilibrium $\left(\hat{N}_{1}^{r}, \hat{N}_{1}^{v}, \hat{N}_{2}^{r}, \hat{N}_{2}^{v}\right)$ is asymptotically stable.

Since on the disease-free subspace $\frac{d}{d \theta} r_{j, k}^{r}\left(\theta, t_{*}\right)=0$ and $\frac{d}{d \theta} r_{j, k}^{v}\left(\theta, t_{*}\right)=0$, from $(I V T)$ we obtain $r_{k, j}^{v}(\tau, t-\tau)=\gamma_{k} R_{k}^{v}(t-\tau)$ and $r_{k, j}^{r}(\tau, t-\tau)=\alpha_{k} R_{k}^{r}(t-\tau)$ for $j, k \in\{1,2\}, j \neq k$. Consider the following subsystem:

$$
\begin{aligned}
& \dot{R}_{j}^{r}(t)=-\left(d_{j}^{r}+\alpha_{j}\right) R_{j}^{r}(t)+\gamma_{k} R_{k}^{v}(t-\tau), \\
& \dot{R}_{j}^{v}(t)=-\left(d_{j}^{v}+\gamma_{j}\right) R_{j}^{v}(t)+\alpha_{k} R_{k}^{r}(t-\tau) .
\end{aligned}
$$

With similar argument as for systems (6) and (7), we obtain that the equilibrium $\left(\hat{R}_{1}^{r}, \hat{R}_{1}^{v}, \hat{R}_{2}^{r}, \hat{R}_{2}^{v}\right)=(0,0,0,0)$ is asymptotically stable. We conclude that $R_{j}^{r}(t), R_{j}^{v}(t) \rightarrow 0$ as $t \rightarrow \infty, j, k \in\{1,2\}, j \neq k$. In the disease-free subspace, $N_{j}^{r}(t)=S_{j}^{r}(t)+R_{j}^{r}(t)$ and $N_{j}^{v}(t)=S_{j}^{v}(t)+R_{j}^{v}(t), j \in\{1,2\}$; thus $S_{j}^{r}(t) \rightarrow \hat{N}_{j}^{r}$ and $S_{j}^{v}(t) \rightarrow \hat{N}_{j}^{v}$ as $t \rightarrow \infty$.

Henceforward, in the disease-free subspace, the solutions of $\left(L^{*}\right)$ converge to the equilibrium $\hat{N}=\left(\hat{N}_{1}^{r}, 0,0,0,0, \hat{N}_{1}^{v}, 0,0,0,0, \hat{N}_{2}^{r}, 0,0,0,0, \hat{N}_{2}^{v}, 0,0,0,0\right)^{T}$.

As an immediate consequence of Propositions 1 and 2, we have that in the disease-free subspace the solutions of $\left(L^{*}\right)$ are bounded.

Proposition 3. If $\delta=0$, then the total populations $\left(N_{1}^{r}(t), N_{1}^{v}(t), N_{2}^{r}(t), N_{2}^{v}(t)\right)$ converge to $\left(\hat{N}_{1}^{r}, \hat{N}_{1}^{v}, \hat{N}_{2}^{r}, \hat{N}_{2}^{v}\right)$, which is given by $(5)$.

Proof. If $\delta=0$, then it is easy to see that $N_{1}^{r}(t), N_{1}^{v}(t), N_{2}^{r}(t), N_{2}^{v}(t)$ satisfy system (4); hence we obtain the same positive equilibrium $\left(\hat{N}_{1}^{r}, \hat{N}_{1}^{v}, \hat{N}_{2}^{r}, \hat{N}_{2}^{v}\right)$, which is globally asymptotically stable.

Proposition 4. Solutions of system $\left(L^{*}\right)$ are bounded. 
Proof. For any $\Phi \in \mathcal{C}_{+}$, the system of $N_{1}^{r}(t), N_{1}^{v}(t), N_{2}^{r}(t), N_{2}^{v}(t)$ becomes

$$
\begin{aligned}
& \dot{N}_{j}^{r}(t)=\Lambda_{j}-\left(d_{j}^{r}+\alpha_{j}\right) N_{j}^{r}(t)-\delta I_{j}^{r}(t)+\gamma_{k} N_{k}^{v}(t-\tau), \\
& \dot{N}_{j}^{v}(t)=-\left(d_{j}^{v}+\gamma_{j}\right) N_{j}^{v}(t)-\delta I_{j}^{v}(t)+\alpha_{k} N_{k}^{r}(t-\tau) .
\end{aligned}
$$

By Proposition $1, N_{j}^{m}(u), I_{j}^{m}(u), j \in\{1,2\}, m \in\{v, r\}$, are nonnegative; thus by a standard comparison argument (see Theorem 4.1 in [13]), solutions of (9) are bounded by the solutions of (4), which are convergent according to Proposition 2. Thus, we conclude that $N_{j}^{r}(t)$ and $N_{j}^{v}(t), j \in\{1,2\}$, are bounded. Since

$$
\begin{aligned}
& 0 \leq S_{j}^{r}(t), E_{j}^{r}(t), A_{j}^{r}(t), I_{j}^{r}(t), R_{j}^{r}(t) \leq N_{j}^{r}(t), \\
& 0 \leq S_{j}^{v}(t), E_{j}^{v}(t), A_{j}^{v}(t), I_{j}^{v}(t), R_{j}^{v}(t) \leq N_{j}^{v}(t),
\end{aligned}
$$

solutions of system $\left(L^{*}\right)$ are bounded.

4. The basic reproduction number. The basic reproduction number $\left(\mathcal{R}_{0}\right)$ is a central quantity in epidemiology, as it determines the average number of secondary infections caused by a typical infected individual during the period of infectiousness. In section 2 we introduced a dynamical model describing the temporal evolution of an infectious disease in and between two regions connected by public transportation. This section is devoted to the computation of the basic (global) reproduction number of the model. It is defined as the dominant eigenvalue of the next generation matrix (NGM), as introduced in $[9,10]$. First we apply some modifications to the model setup and calculate the NGM. Then we show that the reproduction number works as a threshold quantity for the stability of the disease-free equilibrium of the system.

We define the local reproduction numbers as we consider our model in the absence of travel. In this case the two regions are isolated; hence to obtain the (local) reproduction number of region $j, j \in\{1,2\}$, it suffices to follow a typical infected individual during the infectious period in region $j$. Given that the probability of developing symptoms is $p$, the reduction of infectiousness of asymptomatic infecteds is $\rho$, and the average length of the infectious period in classes $I$ and $A$ is $1 / \mu_{I}$ and $1 / \mu_{A}$, respectively, we arrive at the formula

$$
\mathcal{R}_{L, j}=\beta_{j}^{r r}\left(\frac{p}{\mu_{I}}+(1-p) \frac{\rho}{\mu_{A}}\right),
$$

where $\beta_{j}^{r r}$ is the transmission rate in region $j$. In case of isolated regions, the global reproduction number arises as the maximum of the local reproduction numbers. However, the unlimited number of travels and the possibility of disease transmission during travel make it very complicated to trace secondary cases if we incorporate air transportation.

In this section, we neglect the transition from exposed to infected and from infected to recovered classes during travel; i.e., we assume that $\mu_{E}^{T}=\mu_{A}^{T}=\mu_{I}^{T}=0$. Although with this limitation we ignore the possibility of changing to being infectious or recovered on the plane, this assumption also ensures that individuals do not undergo multiple disease states during the same travel. For realistic values of the travel duration $\tau$ it is quite unrealistic to expect that, for instance, someone who was susceptible before travel arrives as recovered upon completing the trip. As shown below, this hypothesis also allows us to calculate the basic reproduction 
number explicitly, and the most important part of the transmission dynamics during travel, namely exposure of susceptibles to the infection, is still fully considered in the modified model.

With the assumption of $\mu_{E}^{T}=\mu_{A}^{T}=\mu_{I}^{T}=0$, our system $(T)$ becomes

$$
\left\{\begin{array}{l}
\frac{d}{d \theta} s_{j, k}^{r}\left(\theta, t_{*}\right)=-s_{j, k}^{r}\left(\theta, t_{*}\right) F_{j, k}^{T}\left(\theta, t_{*}\right), \\
\frac{d}{d \theta} e_{j, k}^{r}\left(\theta, t_{*}\right)=s_{j, k}^{r}\left(\theta, t_{*}\right) F_{j, k}^{T}\left(\theta, t_{*}\right), \\
\frac{d}{d \theta} a_{j, k}^{r}\left(\theta, t_{*}\right)=\frac{d}{d \theta} i_{j, k}^{r}\left(\theta, t_{*}\right)=\frac{d}{d \theta} r_{j, k}^{r}\left(\theta, t_{*}\right)=0, \\
\frac{d}{d \theta} s_{j, k}^{v}\left(\theta, t_{*}\right)=-s_{j, k}^{v}\left(\theta, t_{*}\right) F_{j, k}^{T}\left(\theta, t_{*}\right), \\
\frac{d}{d \theta} e_{j, k}^{v}\left(\theta, t_{*}\right)=s_{j, k}^{v}\left(\theta, t_{*}\right) F_{j, k}^{T}\left(\theta, t_{*}\right), \\
\frac{d}{d \theta} a_{j, k}^{v}\left(\theta, t_{*}\right)=\frac{d}{d \theta} i_{j, k}^{v}\left(\theta, t_{*}\right)=\frac{d}{d \theta} r_{j, k}^{v}\left(\theta, t_{*}\right)=0,
\end{array}\right.
$$

with

$$
\begin{aligned}
& F_{j, k}^{T}\left(\theta, t_{*}\right)=\frac{\beta^{T}}{n_{j, k}\left(\theta, t_{*}\right)}\left(i_{j, k}^{r}\left(\theta, t_{*}\right)+i_{j, k}^{v}\left(\theta, t_{*}\right)+\rho\left(a_{j, k}^{r}\left(\theta, t_{*}\right)+a_{j, k}^{v}\left(\theta, t_{*}\right)\right)\right), \\
& n_{j, k}\left(\theta, t_{*}\right)=\alpha_{j} N_{j}^{r}\left(t_{*}\right)+\gamma_{j} N_{j}^{v}\left(t_{*}\right)
\end{aligned}
$$

for $j, k \in\{1,2\}, j \neq k$. Using systems ( $\left.T^{\prime}\right)$ and (IVT), we obtain the densities of asymptomatic, symptomatic infected, and recovered individuals during travel with respect to $\theta$ as

$$
\begin{array}{ll}
a_{j, k}^{r}\left(\theta, t_{*}\right)=a_{j, k}^{r}\left(0, t_{*}\right)=\alpha_{j} A_{j}^{r}\left(t_{*}\right), & a_{j, k}^{v}\left(\theta, t_{*}\right)=a_{j, k}^{v}\left(0, t_{*}\right)=\gamma_{j} A_{j}^{v}\left(t_{*}\right), \\
i_{j, k}^{r}\left(\theta, t_{*}\right)=i_{j, k}^{r}\left(0, t_{*}\right)=\alpha_{j} I_{j}^{r}\left(t_{*}\right), & i_{j, k}^{v}\left(\theta, t_{*}\right)=i_{j, k}^{v}\left(0, t_{*}\right)=\gamma_{j} I_{j}^{v}\left(t_{*}\right), \\
r_{j, k}^{r}\left(\theta, t_{*}\right)=r_{j, k}^{r}\left(0, t_{*}\right)=\alpha_{j} R_{j}^{r}\left(t_{*}\right), & r_{j, k}^{v}\left(\theta, t_{*}\right)=r_{j, k}^{v}\left(0, t_{*}\right)=\gamma_{j} R_{j}^{v}\left(t_{*}\right)
\end{array}
$$

for all $t_{*}, \theta \in[0, \tau]$, and $j, k \in\{1,2\}, j \neq k$. Then, using (10), the force of infection $F_{j, k}^{T}$ arises as

$$
F_{j, k}^{T}\left(\theta, t_{*}\right)=\beta^{T} \frac{\alpha_{j} I_{j}^{r}\left(t_{*}\right)+\gamma_{j} I_{j}^{v}\left(t_{*}\right)+\rho\left(\alpha_{j} A_{j}^{r}\left(t_{*}\right)+\gamma_{j} A_{j}^{v}\left(t_{*}\right)\right)}{\alpha_{j} N_{j}^{r}\left(t_{*}\right)+\gamma_{j} N_{j}^{v}\left(t_{*}\right)},
$$

where $\theta \in[0, \tau]$ and $j, k \in\{1,2\}, j \neq k$, and we can determine the density of susceptible individuals during travel for $\theta \in[0, \tau], j, k \in\{1,2\}, j \neq k$, as

$$
\begin{aligned}
s_{j, k}^{r}\left(\theta, t_{*}\right) & =s_{j, k}^{r}\left(0, t_{*}\right) e^{-\int_{0}^{\theta} F_{j, k}^{T}\left(\nu, t_{*}\right) d \nu} \\
& =\alpha_{j} S_{j}^{r}\left(t_{*}\right) e^{-\theta \beta^{T} \frac{\alpha_{j} I_{j}^{r}\left(t_{*}\right)+\gamma_{j} I_{j}^{v}\left(t_{*}\right)+\rho\left(\alpha_{j} A_{j}^{r}\left(t_{*}\right)+\gamma_{j} A_{j}^{v}\left(t_{*}\right)\right)}{\alpha_{j} N_{j}^{r}\left(t_{*}\right)+\gamma_{j} N_{j}^{v}\left(t_{*}\right)}}, \\
s_{j, k}^{v}\left(\theta, t_{*}\right) & =s_{j, k}^{v}\left(0, t_{*}\right) e^{-\int_{0}^{\theta} F_{j, k}^{T}\left(\nu, t_{*}\right) d \nu} \\
& =\gamma_{j} S_{j}^{v}\left(t_{*}\right) e^{-\theta \beta^{T} \frac{\alpha_{j} I_{j}^{r}\left(t_{*}\right)+\gamma_{j} I_{j}^{v}\left(t_{*}\right)+\rho\left(\alpha_{j} A_{j}^{r}\left(t_{*}\right)+\gamma_{j} A_{j}^{v}\left(t_{*}\right)\right)}{\alpha_{j} N_{j}^{r}\left(t_{*}\right)+\gamma_{j} N_{j}^{v}\left(t_{*}\right)}} .
\end{aligned}
$$


Last, using the definition of $n_{j, k}^{m}\left(\theta, t_{*}\right)(j, k \in\{1,2\}, m \in\{r, v\})$, we obtain the density of exposed individuals during travel as

$$
\begin{aligned}
e_{j, k}^{r}\left(\theta, t_{*}\right)= & n_{j, k}^{r}\left(\theta, t_{*}\right)-a_{j, k}^{r}\left(\theta, t_{*}\right)-i_{j, k}^{r}\left(\theta, t_{*}\right)-r_{j, k}^{r}\left(\theta, t_{*}\right)-s_{j, k}^{r}\left(\theta, t_{*}\right) \\
= & \alpha_{j} N_{j}^{r}\left(t_{*}\right)-\alpha_{j} A_{j}^{r}\left(t_{*}\right)-\alpha_{j} I_{j}^{r}\left(t_{*}\right)-\alpha_{j} R_{j}^{r}\left(t_{*}\right)-s_{j, k}^{r}\left(\theta, t_{*}\right) \\
= & \alpha_{j}\left(S_{j}^{r}\left(t_{*}\right)\left(1-e^{-\theta \beta^{T} \frac{\alpha_{j} I_{j}^{r}\left(t_{*}\right)+\gamma_{j} I_{j}^{v}\left(t_{*}\right)+\rho\left(\alpha_{j} A_{j}^{r}\left(t_{*}\right)+\gamma_{j} A_{j}^{v}\left(t_{*}\right)\right)}{\alpha_{j} N_{j}^{*}\left(t_{*}\right)+\gamma_{j} N_{j}^{v}\left(t_{*}\right)}}\right)+E_{j}^{r}\left(t_{*}\right)\right), \\
e_{j, k}^{v}\left(\theta, t_{*}\right)= & n_{j, k}^{v}\left(\theta, t_{*}\right)-a_{j, k}^{v}\left(\theta, t_{*}\right)-i_{j, k}^{v}\left(\theta, t_{*}\right)-r_{j, k}^{v}\left(\theta, t_{*}\right)-s_{j, k}^{v}\left(\theta, t_{*}\right) \\
= & \gamma_{j} N_{j}^{v}\left(t_{*}\right)-\gamma_{j} A_{j}^{v}\left(t_{*}\right)-\gamma_{j} I_{j}^{v}\left(t_{*}\right)-\gamma_{j} R_{j}^{v}\left(t_{*}\right)-s_{j, k}^{v}\left(\theta, t_{*}\right) \\
= & \gamma_{j}\left(S_{j}^{v}\left(t_{*}\right)+E_{j}^{v}\left(t_{*}\right)\right) \\
& -\gamma_{j} S_{j}^{v}\left(t_{*}\right) e^{-\theta \beta^{T} \frac{\alpha_{j} I_{j}^{r}\left(t_{*}\right)+\gamma_{j} r_{j}^{v}\left(t_{*}\right)+\rho\left(\alpha_{j} A_{j}^{r}\left(t_{*}\right)+\gamma_{j} A_{j}^{v}\left(t_{*}\right)\right)}{\alpha_{j} N_{j}^{r}\left(t_{*}\right)+\gamma_{j} N_{j}^{v}\left(t_{*}\right)}} .
\end{aligned}
$$

Choosing $\theta=\tau$ and $t_{*}=t-\tau$, the inflow terms $s_{j, k}^{m}(\tau, t-\tau), e_{j, k}^{m}(\tau, t-\tau), a_{j, k}^{m}(\tau, t-\tau)$, $i_{j, k}^{m}(\tau, t-\tau)$, and $r_{j, k}^{m}(\tau, t-\tau)(j, k \in\{1,2\}, m \in\{r, v\})$ determined in (10), (11), and (12) arise as delay terms of $S_{j}^{m}, E_{j}^{m}, A_{j}^{m}, I_{j}^{m}$, and $R_{j}^{m}$. In what follows, we use the notation $\left(L^{\prime}\right)$ to refer to the special case of $(L)$ with these particular inflow terms.

We use the notation of section 3 and define $\bar{H}: \mathbb{R}^{20} \rightarrow \mathbb{R}^{20}$ as $\bar{H}_{i}$ equals the inflow term of the right-hand side of the equation for $X_{i}$ in $\left(L^{\prime}\right)$. For instance,

$$
\bar{H}_{1}(Y)=\gamma_{2} Y_{16} e^{-\tau \beta^{T} \frac{\alpha_{2} Y_{14}+\gamma_{2} Y_{19}+\rho\left(\alpha_{2} Y_{13}+\gamma_{2} Y_{18}\right)}{\alpha_{2} \sum_{j=11}^{15} Y_{j}+\gamma_{2} \sum_{j=16}^{20} Y_{j}}}, \quad \bar{H}_{18}(Y)=\alpha_{1} Y_{3} .
$$

System $\left(L^{\prime}\right)$ can be written in the compact form of

$$
\dot{X}(t)=\overline{\mathcal{F}}(X(t), X(t-\tau)),
$$

where $\overline{\mathcal{F}}(X(t), X(t-\tau))=G(X(t))+\bar{H}(X(t-\tau)), \overline{\mathcal{F}}: \mathbb{R}^{20} \times \mathbb{R}^{20} \rightarrow \mathbb{R}^{20}$. Now we focus on system $(\bar{L})$, and we detail the computation of the reproduction number.

Notice that the disease-free equilibrium $\hat{N}=\left(\hat{N}_{1}^{r}, 0,0,0,0, \hat{N}_{1}^{v}, 0,0,0,0, \hat{N}_{2}^{r}, 0,0,0,0, \hat{N}_{2}^{v}, 0,0,0,0\right)$ of system $\left(L^{*}\right)$ defined in section 3 is the unique positive equilibrium of $(\bar{L})$ in the disease-free subspace. In the initial stage of the epidemic, we can assume that system $(\bar{L})$ is near the equilibrium $\hat{N}$ and approximate the equations of classes $E_{j}^{m}, A_{j}^{m}, I_{j}^{m}, j \in\{1,2\}, m \in\{r, v\}$, with the linear system

$$
z^{\prime}(t)=\mathcal{A} z(t)+\mathcal{B} z(t-\tau)
$$

where $z: \mathbb{R} \mathbb{R}^{12}, \mathcal{A}, \mathcal{B} \in \mathbb{R}^{12 \times 12}$ and $\mathcal{A}=D G(\hat{N}), \mathcal{B}=D \bar{H}(\hat{N})$ hold. Matrices $\mathcal{A}$ and $\mathcal{B}$ have the form

$$
\mathcal{A}=\left(\begin{array}{cccc}
A_{11} & A_{12} & A_{13} & A_{14} \\
A_{21} & A_{22} & A_{23} & A_{24} \\
A_{31} & A_{32} & A_{33} & A_{34} \\
A_{41} & A_{42} & A_{43} & A_{44}
\end{array}\right), \quad \mathcal{B}=\left(\begin{array}{llll}
B_{11} & B_{12} & B_{13} & B_{14} \\
B_{21} & B_{22} & B_{23} & B_{24} \\
B_{31} & B_{32} & B_{33} & B_{34} \\
B_{41} & B_{42} & B_{43} & B_{44}
\end{array}\right)
$$


where $A_{j, k}, B_{j, k} \in \mathbb{R}^{3 \times 3}$ for $j, k \in\{1,2,3,4\}$, and $A_{j, k}$ and $B_{j, k}$ can be obtained as follows:

$$
\begin{gathered}
A_{11}=\left(\begin{array}{ccc}
-\left(\mu_{E}+\alpha_{1}+d_{1}^{r}\right) & \rho \beta_{1}^{r r} \frac{\hat{N}_{1}^{r}}{\hat{N}_{1}^{r}+\hat{N}_{1}^{v}} & \beta_{1}^{r r} \frac{\hat{N}_{1}^{r}}{\hat{N}_{1}^{r}+\hat{N}_{1}^{v}} \\
(1-p) \mu_{E} & -\left(\mu_{A}+\alpha_{1}+d_{1}^{r}\right) & 0 \\
p \mu_{E} & 0 & -\left(\mu_{I}+\alpha_{1}+\delta+d_{1}^{r}\right)
\end{array}\right), \\
A_{12}=\left(\begin{array}{ccc}
0 & \rho \beta_{1}^{v r} \frac{\hat{N}_{1}^{r}}{\hat{N}_{1}^{r}+\hat{N}_{1}^{v}} & \beta_{1}^{v r} \frac{\hat{N}_{1}^{r}}{\hat{N}_{1}^{r}+\hat{N}_{1}^{v}} \\
0 & 0 & 0 \\
0 & 0 & 0
\end{array}\right),
\end{gathered}
$$

and $A_{1,3}=A_{1,4}=\mathcal{O}$, where we denote the matrix with 0 -entries by $\mathcal{O}$; moreover,

$$
B_{13}=\left(\begin{array}{ccc}
0 & \frac{\tau \beta^{T} \rho \alpha_{2} \gamma_{2} \hat{N}_{2}^{v}}{\alpha_{2} \hat{N}_{2}^{r}+\gamma_{2} \hat{N}_{2}^{v}} & \frac{\tau \beta^{T} \alpha_{2} \gamma_{2} \hat{N}_{2}^{v}}{\alpha_{2} \hat{N}_{2}^{r}+\gamma_{2} \hat{N}_{2}^{v}} \\
0 & 0 & 0 \\
0 & 0 & 0
\end{array}\right), \quad B_{14}=\left(\begin{array}{ccc}
\gamma_{2} & \frac{\tau \beta^{T} \rho \gamma_{2}^{2} \hat{N}_{2}^{v}}{\alpha_{2} \hat{N}_{2}^{r}+\gamma_{2} \hat{N}_{2}^{v}} & \frac{\tau \beta^{T} \gamma_{2}^{2} \hat{N}_{2}^{v}}{\alpha_{2} \hat{N}_{2}^{r}+\gamma_{2} \hat{N}_{2}^{v}} \\
0 & \gamma_{2} & 0 \\
0 & 0 & \gamma_{2}
\end{array}\right),
$$

and $B_{1,1}=B_{1,2}=\mathcal{O}$. Matrix elements $A_{j, k}, B_{j, k}, j \in\{2,3,4\}, k \in\{1,2,3,4\}$, can be derived similarly.

Next we decompose the matrix $\mathcal{A}+\mathcal{B}$ as $\mathcal{F}-\mathcal{V}$, where $\mathcal{F}$ is the transmission part, describing the production of new infections, and $-\mathcal{V}$ is the transition part, describing changes in state (see $[9,34]$ for some details). To do that, we first determine what we call reproduction here, i.e., in what kind of situations do new infections occur. We define two possible ways of reproduction:

(i) a susceptible moves to the exposed class while being in a region;

(ii) an exposed individual, who was susceptible before travel, arrives to a region upon completing a trip.

With this definition in mind, we obtain $\mathcal{F} \in \mathbb{R}^{12 \times 12}$ and $\mathcal{V} \in \mathbb{R}^{12 \times 12}$ as

$$
\mathcal{F}=\left(\begin{array}{cccc}
F_{11} & F_{12} & F_{13} & F_{14} \\
F_{21} & F_{22} & F_{23} & F_{24} \\
F_{31} & F_{32} & F_{33} & F_{34} \\
F_{41} & F_{42} & F_{43} & F_{44}
\end{array}\right), \quad \mathcal{V}=\left(\begin{array}{llll}
V_{11} & V_{12} & V_{13} & V_{14} \\
V_{21} & V_{22} & V_{23} & V_{24} \\
V_{31} & V_{32} & V_{33} & V_{34} \\
V_{41} & V_{42} & V_{43} & V_{44}
\end{array}\right),
$$

where $F_{j, k}, V_{j, k} \in \mathbb{R}^{3 \times 3}(j, k \in\{1,2,3,4\})$. It is easy to see that

$$
\begin{aligned}
& F_{11}=\left(\begin{array}{cccc}
0 & \rho \beta_{1}^{r r} \frac{\hat{N}_{1}^{r}}{\hat{N}_{1}^{r}+\hat{N}_{1}^{v}} & \beta_{1}^{r r} \frac{\hat{N}_{1}^{r}}{\hat{N}_{1}^{r}+\hat{N}_{1}^{v}} \\
0 & 0 & 0 \\
0 & 0 & 0
\end{array}\right), \quad F_{12}=\left(\begin{array}{ccc}
0 & \rho \beta_{1}^{v r} \frac{\hat{N}_{1}^{r}}{\hat{N}_{1}^{r}+\hat{N}_{1}^{v}} & \beta_{1}^{v r} \frac{\hat{N}_{1}^{r}}{\hat{N}_{1}^{r}+\hat{N}_{1}^{v}} \\
0 & 0 & 0 \\
0 & 0 & 0
\end{array}\right), \\
& F_{13}=\left(\begin{array}{ccc}
0 & \frac{\tau \beta^{T} \rho \alpha_{2} \gamma_{2} \hat{N}_{2}^{v}}{\alpha_{2} \hat{N}_{2}^{r}+\gamma_{2} \hat{N}_{2}^{v}} & \frac{\tau \beta^{T} \alpha_{2} \gamma_{2} \hat{N}_{2}^{v}}{\alpha_{2} \hat{N}_{2}^{r}+\gamma_{2} \hat{N}_{2}^{v}} \\
0 & 0 & 0 \\
0 & 0 & 0
\end{array}\right), \quad F_{14}=\left(\begin{array}{ccc}
0 & \frac{\tau \beta^{T} \rho \gamma_{2}^{2} \hat{N}_{2}^{v}}{\alpha_{2} \hat{N}_{2}^{r}+\gamma_{2} \hat{N}_{2}^{v}} & \frac{\tau \beta^{T} \gamma_{2}^{2} \hat{N}_{2}^{v}}{\alpha_{2} \hat{N}_{2}^{r}+\gamma_{2} \hat{N}_{2}^{v}} \\
0 & 0 & 0 \\
0 & 0 & 0
\end{array}\right)
\end{aligned}
$$


and elements $F_{j, k}, j \in\{2,3,4\}, k \in\{1,2,3,4\}$, arise similarly. The elements of $-\mathcal{V}$ represent rates at which individuals progress from one class to another:

$V_{11}=\left(\begin{array}{ccc}\mu_{E}+\alpha_{1}+d_{1}^{r} & 0 & 0 \\ -(1-p) \mu_{E} & \mu_{A}+\alpha_{1}+d_{1}^{r} & 0 \\ -p \mu_{E} & 0 & \mu_{I}+\alpha_{1}+\delta+d_{1}^{r}\end{array}\right), \quad V_{14}=\left(\begin{array}{ccc}-\gamma_{2} & 0 & 0 \\ 0 & -\gamma_{2} & 0 \\ 0 & 0 & -\gamma_{2}\end{array}\right)$,

while $V_{1,2}=V_{1,3}=\mathcal{O}$ and elements $V_{j, k}, j \in\{2,3,4\}, k \in\{1,2,3,4\}$, can be obtained similarly. Clearly $\mathcal{F}$ is a positive matrix, that is, all of its entries are nonnegative, and it is easy to check that $-\mathcal{V}$ is positive-off-diagonal, that is, all entries are nonnegative except possibly those on the diagonal. For a square matrix $M$ we define the spectral bound $s(M)$ and the spectral radius $\rho(M)$ by $s(M):=\sup \{\operatorname{Re}(\lambda): \lambda \in \sigma(M)\}, \rho(M):=\sup \{|\lambda|: \lambda \in \sigma(M)\}$, where $\sigma(M)$ denotes the set of eigenvalues of $M$. One can show that $s(-\mathcal{V})<0$; this is equivalent to the statement that $\mathcal{V}$ is invertible and $\mathcal{V}^{-1}$ is a positive matrix (for the proof of the equivalence, see, e.g., Lemma 6.12 of [9]). We state the following proposition.

Proposition 5. The zero solution of the linear delay differential equation

$$
z^{\prime}(t)=\mathcal{A} z(t)+\mathcal{B} z(t-\tau)
$$

is asymptotically stable if $\rho\left(\mathcal{F} \mathcal{V}^{-1}\right)<1$ and unstable if $\rho\left(\mathcal{F} \mathcal{V}^{-1}\right)>1$.

Proof. The principal result of section 5 in Chapter 5 in [28] is that the stability of an equilibrium of a cooperative and irreducible system of delay differential equations is the same as that for an associated system of cooperative ordinary differential equations. System (13) is cooperative since $\mathcal{A}$ is positive-off-diagonal and $\mathcal{B}$ is a positive matrix. Every column of $\mathcal{B}$ contains at least one nonzero element, which together with the irreducibility of matrix $\mathcal{A}+\mathcal{B}$ implies that system (13) is irreducible. Corollary 5.2 in [28] states that the zero solution of the linear delay differential equation (13) is asymptotically stable (unstable) if and only if the zero solution of the linear ordinary differential equation

$$
w^{\prime}(t)=(\mathcal{A}+\mathcal{B}) w(t)
$$

is asymptotically stable (unstable). We can reformulate (14) as

$$
w^{\prime}(t)=(\mathcal{F}-\mathcal{V}) w(t) .
$$

We have seen that $\mathcal{F}$ is a positive matrix and $-\mathcal{V}$ is a positive-off-diagonal matrix with $s(-\mathcal{V})<0$. The stability of the zero steady state of $w^{\prime}(t)=(\mathcal{F}-\mathcal{V}) w(t)$ is determined by the sign of $s(\mathcal{F}-\mathcal{V})$, which coincides with the sign of $\rho\left(\mathcal{F} \mathcal{V}^{-1}\right)-1$ (see Theorem A.1 in [11]). The proof is now complete.

The statement of Proposition 5 extends to the nonlinear system $(\bar{L})$ by the principle of linearized stability.

Proposition 6. The disease-free equilibrium of system $(\bar{L})$ is asymptotically stable if $\rho\left(\mathcal{F} \mathcal{V}^{-1}\right)<$ 1 , and unstable if $\rho\left(\mathcal{F} \mathcal{V}^{-1}\right)>1$.

After obtaining stability results for $(\bar{L})$, a system of delay differential equations, we consider the following associated system of ODEs:

$$
\dot{X}(t)=G(X(t))+\bar{H}(X(t)),
$$


where $\bar{H}$ was introduced previously in this section and $X$ and $G$ were defined in section 3 . The concept of the next generation matrix (NGM) of an epidemic model was introduced in Diekmann, Heesterbeck, and Metz [10] (and elaborated in [9, Chapter 5]) as a matrix whose elements give the number of newly infected individuals in specific categories. To obtain this matrix, one considers the equations of the system that describe the production of new infections and changes in state among infected individuals; in case of system (15), this infected subsystem consists of the equations for $E_{j}^{m}, A_{j}^{m}, I_{j}^{m}, j \in\{1,2\}, m \in\{r, v\}$. Clearly $\hat{N}$ works as the unique disease-free equilibrium of (15), so we can linearize the infected subsystem about the infection-free steady state and get

$$
w^{\prime}(t)=(\mathcal{A}+\mathcal{B}) w(t)=(\mathcal{F}-\mathcal{V}) w(t) .
$$

Diekmann, Heesterbeck, and Robert [11] refers to $\mathcal{F} \mathcal{V}^{-1} \in \mathbb{R}^{12 \times 12}$ as $\mathcal{K}_{L}$, the NEM for system (15) with large domain. However, this matrix does not equal the NGM $\mathcal{K}$ of the ODE system (15), because the decomposition of $\mathcal{A}+\mathcal{B}$ relates to the expected offspring of individuals of any state and not just epidemiological newborns (new infections). Since in the case of system (15) only states $E_{1}^{r}, E_{1}^{v}, E_{2}^{r}$, and $E_{2}^{v}$ are involved in the action of the NGM $\mathcal{K}$, it is clear that $\mathcal{K} \in \mathbb{R}^{4 \times 4}$. [11] claims that $\rho\left(\mathcal{K}_{L}\right)=\rho(\mathcal{K})$, and it can be shown that for (15) the NGM can be obtained as $\mathcal{K}=\left(\mathcal{K}_{L}\right)_{1,4,7,10}^{1,4,7,10}$.

Next we determine the NGM $\mathcal{N}$ and the reproduction number $\mathcal{R}_{0}$ for the delay system $(\bar{L})$. Then we show that $\mathcal{N}=\mathcal{K}$; i.e., the NGM for the delay system $(\bar{L})$ equals NGM for the ODE system (15). Since $\mathcal{R}_{0}$ is defined as the dominant eigenvalue of $\mathcal{N}$ (the existence of the dominant eigenvalue is guaranteed by the Frobenius-Perron theorem), and, moreover, $K_{L}=\mathcal{F} \mathcal{V}^{-1}$ and $\rho\left(\mathcal{K}_{L}\right)=\rho(\mathcal{K})$ hold, we obtain that $\mathcal{R}_{0}=\rho\left(\mathcal{F} \mathcal{V}^{-1}\right)$. If so, then it follows from Proposition 6 that $\mathcal{R}_{0}$ works as a threshold quantity for the stability of the disease-free equilibrium of system $(\bar{L})$.

We construct the NGM $\mathcal{N}$ for system $(\bar{L})$ as we divide all exposed individuals into four groups: residents of region $1\left(E_{1}^{r}\right)$, visitors of region $1\left(E_{1}^{v}\right)$, residents of region $2\left(E_{2}^{r}\right)$, and visitors of region $2\left(E_{2}^{v}\right)$. We denote the number of new infections among individuals of region $k$ with residential status $n$ generated by an exposed individual of region $j$ with residential status $m$ by $R_{j, k}^{m, n}$, where $j, k \in\{1,2\}, m, n \in\{r, v\}$. Then $\mathcal{N} \in \mathbb{R}^{4 \times 4}$ has the form

$$
\mathcal{N}=\left(\begin{array}{llll}
R_{11}^{r r} & R_{11}^{v r} & R_{21}^{r r} & R_{21}^{v r} \\
R_{11}^{r v} & R_{11}^{v v} & R_{21}^{r v} & R_{21}^{v v} \\
R_{12}^{r r} & R_{12}^{v r} & R_{22}^{r r} & R_{22}^{v r} \\
R_{12}^{r v} & R_{12}^{v v} & R_{22}^{r v} & R_{22}^{v v}
\end{array}\right)
$$

We can obtain the elements of $\mathcal{N}$ by biological reasoning, directly from the specification of the system, and using our definition of reproduction. For the calculations (from which it follows that $\mathcal{N}=\mathcal{K})$, see the appendix.

4.1. The dependence of $\mathcal{R}_{0}$ on key model parameters. Throughout this subsection we demonstrate how the reproduction number depends on various key model parameters. We previously described the calculation of $\mathcal{R}_{0}$ as the dominant eigenvalue of the NGM. We obtained that each element of this $4 \times 4$ matrix arises as a complex formula of the model 


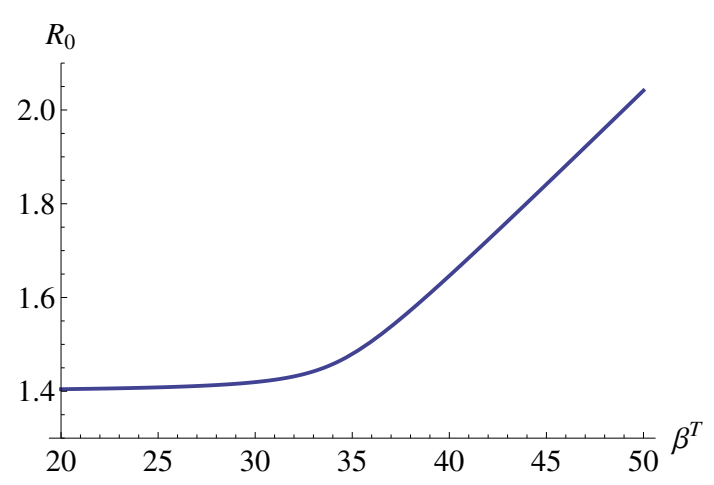

Figure 2. The dependence of $\mathcal{R}_{0}$ on $\beta^{T}$. Parameter values were chosen as $\mathcal{R}_{L, 1}=1.4, \mathcal{R}_{L, 2}=1.4, \tau=0.5$, $\gamma_{1}^{-1}=\gamma_{2}^{-1}=7, \mu_{E}^{-1}=1.4, \mu_{I}^{-1}=3, \mu_{A}^{-1}=4.1, p=0.6, \rho=0.1$.

parameters (see the appendix for details); hence due to the complicated structure, here we present not analytic results but numerical simulations with reasonable parameter values. We performed a systematic analysis to reveal the dependence of $\mathcal{R}_{0}$ on several model parameters. We chose reasonable values for the parameters (see section 5 for plausible parameter ranges for influenza), population sizes, and travel rates (see [2]). In this subsection we assume that the two regions are symmetric in the population sizes, travel rates, and epidemiological characteristics. This assumption enables us to focus on a better understanding of the role of the key parameters. The findings support our intuitions about the dependence of the basic reproduction number on epidemiological parameters like the transmission rates and duration of infectious periods: increasing the values of these parameters results in an increase of the value of the reproduction number. However, we observed some unexpected behavior when examining $\mathcal{R}_{0}$ as a function of the transmission rate during travel and the travel rate of visitors.

Figure 2 shows $\mathcal{R}_{0}$ as a function of $\beta^{T}$ when other model parameters are fixed and the local reproduction numbers are set to 1.4. For $\beta^{T}<30$ the reproduction number settles at around 1.4; however, the function approaches a line with a strictly positive slope as we further increase the parameter value. Numerical simulations proved that there is an eigenvalue of the NGM whose dependence on $\beta^{T}$ is almost linear. As the parameter value grows, this eigenvalue dominates the one which is close to 1.4 for each value of $\beta^{T}$. Next we set $\beta^{T}=40$ and examine the effect of parameters $\gamma_{1}$ and $\gamma_{2}$ on the reproduction number. If we ignore the time needed to complete a one-way travel, the value of these parameters does not influence the value of $\mathcal{R}_{0}$ in the case of identical regions: if $\tau=0$ and the two regions are symmetric in the population sizes and values of epidemiological parameters including the local reproduction numbers, then $\mathcal{R}_{0}=\mathcal{R}_{L, 1}=\mathcal{R}_{L, 2}$ holds. However, for positive values of $\tau$ we obtain some nonmonotonic behavior of $\mathcal{R}_{0}$ as a function of the duration of visitors' stay (reciprocal of $\gamma_{1}$ and $\gamma_{2}$ ), as shown in Figure 3. To understand this phenomenon we examined how the elements of the NGM depend on $\frac{1}{\gamma_{1}}$ and $\frac{1}{\gamma_{2}}$. We found that these parameters do not significantly influence most of the matrix elements if one considers realistic parameter range $\left(0<\frac{1}{\gamma_{1}}, \frac{1}{\gamma_{2}}<50\right.$ (days)). However, $R_{21}^{v r}, R_{21}^{v v}, R_{12}^{v r}$, and $R_{12}^{v v}$ have similar nonmonotonic shapes as obtained by the reproduction number. 


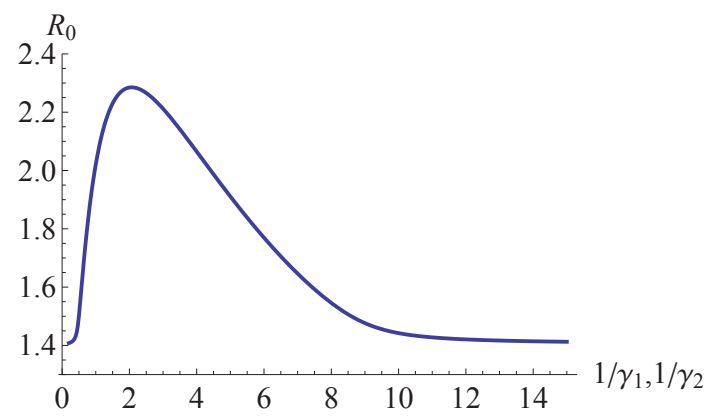

Figure 3. The dependence of $\mathcal{R}_{0}$ on $\frac{1}{\gamma_{1}}$ and $\frac{1}{\gamma_{2}}$ in the case of $\gamma_{1}=\gamma_{2}$ and $\tau>0$. Parameter values were chosen as $\mathcal{R}_{L, 1}=1.4, \mathcal{R}_{L, 2}=1.4, \tau=0.5, \beta^{T}=40, \mu_{E}^{-1}=1.4, \mu_{I}^{-1}=3, \mu_{A}^{-1}=4.1, p=0.6, \rho=0.1$.

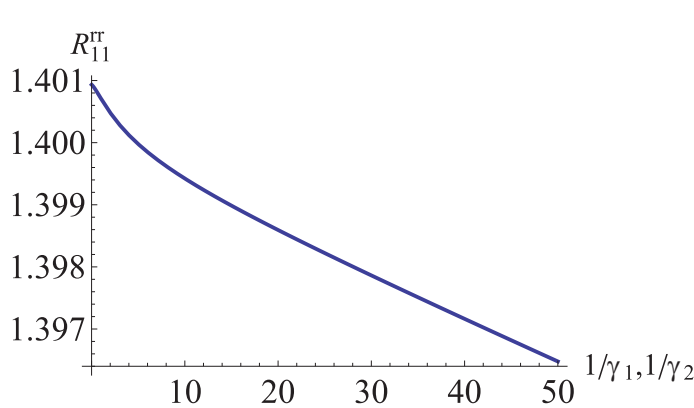

(a) $R_{11}^{r r}$

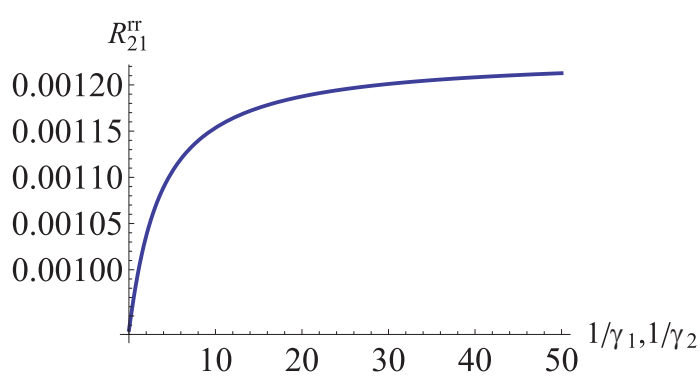

(c) $R_{21}^{r r}$

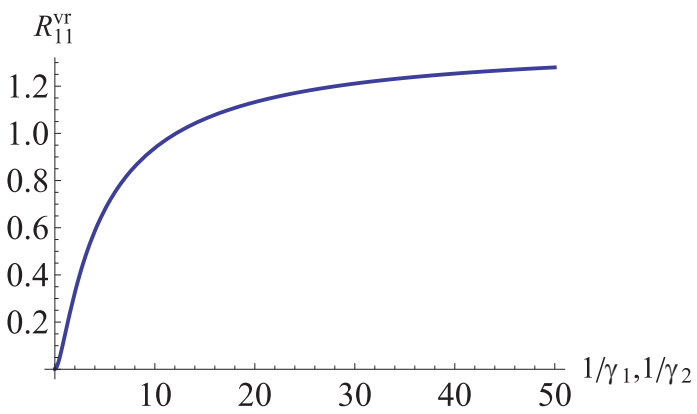

(b) $R_{11}^{v r}$

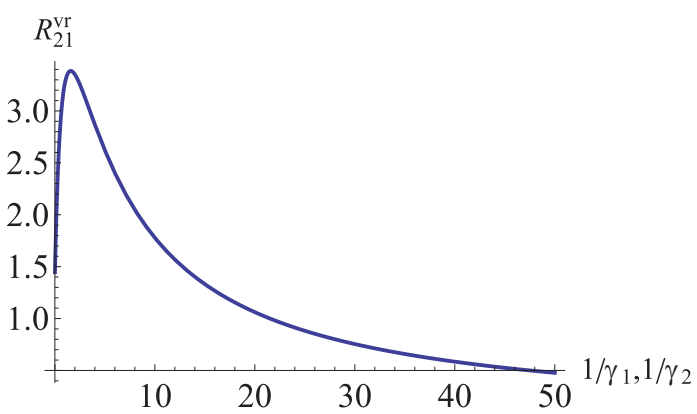

(d) $R_{21}^{v r}$

Figure 4. The dependence of some elements of the next generation matrix on $\frac{1}{\gamma_{1}}$ and $\frac{1}{\gamma_{2}}$ in the case of $\gamma_{1}=\gamma_{2}$ and $\tau>0$. Parameter values were chosen as $\mathcal{R}_{L, 1}=1.4, \mathcal{R}_{L, 2}=1.4, \tau=0.5, \beta^{T}=40, \mu_{E}^{-1}=1.4$, $\mu_{I}^{-1}=3, \mu_{A}^{-1}=4.1, p=0.6, \rho=0.1$.

See Figure 4 for the graph of four elements of the NGM. The values of $R_{11}^{r r}$ and $R_{21}^{r r}$ do not depend strongly on the length of visitors' stay, as for real air traffic data travel rates of residents are low; hence with high probability the exposed resident never becomes a visitor. The longer visitors stay in the foreign region on average, the higher $R_{11}^{v r}$ is (it converges to $\mathcal{R}_{L, 1}$ as $\frac{1}{\gamma_{1}}$ and $\frac{1}{\gamma_{2}}$ tend to infinity). The element $R_{21}^{v r}$ defines the number of new infections among residents of region 1 caused by a single exposed visitor of region 2. Following our 


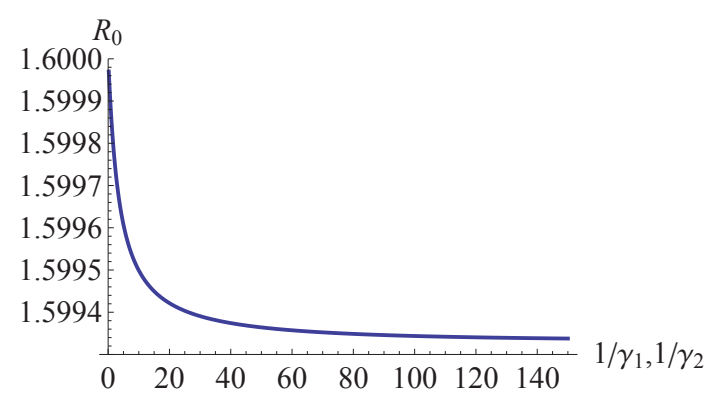

Figure 5. The dependence of $\mathcal{R}_{0}$ on $\frac{1}{\gamma_{1}}$ and $\frac{1}{\gamma_{2}}$ in the case of $\gamma_{1}=\gamma_{2}$ and $\tau=0$; i.e., we neglect the

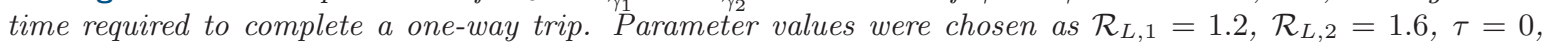
$\mu_{E}^{-1}=1.4, \mu_{I}^{-1}=3, \mu_{A}^{-1}=4.1, p=0.6, \rho=0.1$.

definition of reproduction, $R_{21}^{v r}$ counts new infections accrued while the visitor was traveling to region 1 and while the visitor was staying there. If the duration of visitors' stay is short, the probability that a visitor leaves region 2 before finishing his exposed period is high, and as we neglected the possibility of moving to classes $I$ or $A$ during travel, he will start his infectious period only in region 1 . Choosing realistic values for travel rates makes the chance of leaving region 1 small; thus, for small values of $\frac{1}{\gamma_{1}}$ and $\frac{1}{\gamma_{2}}, R_{21}^{v r}$ approximately equals 1.4 , the value of the local reproduction number $\mathcal{R}_{L, 1}$. As the length of visitors' stay increases, the chances for the visitor to move to class $I$ or $A$ before traveling back to region 1 rise. This results in the elevated number of newly infected individuals due to increased transmission potential during travel. However, if the duration of visitors' stay is much longer, the probability that the visitor travels back to region 1 and gets into contact with residents there (in region 1) is low; hence the expected number of such new infections is close to 0 . The graphical interpretation of other elements of the NGM can be explained similarly. We remark that the nonmonotonic behavior of the reproduction number as a function of the duration of visitors' stay is observed in the case of asymmetric regions as well. However, if we neglect the duration of travel and assume different local reproduction numbers in the regions, the graph of $\mathcal{R}_{0}$ becomes monotonically decreasing, as shown in Figure 5. This shows that including travel-related infections can fundamentally change the way the reproduction number depends on various model parameters, and in the case of a nonmonotone dependence on a parameter, one has to be very careful when proposing control measures which change the given parameter.

4.2. $\mathcal{R}_{0}$ as the threshold quantity for epidemic outbreaks. The importance of parameter $\beta^{T}$ in the computation of the reproduction number has been revealed in the previous subsection. However, it is not clear how the nonlinear dependence of $\mathcal{R}_{0}$ on the transmission rate during travel is reflected on the epidemic curves or the final epidemic size. We consider the hypothetical case when the two regions are symmetric in the population sizes, travel rates, and values of every model parameter except the local reproduction numbers $\left(\mathcal{R}_{L, 1}=\mathcal{R}_{L, 2}\right.$ would result in identical epidemic curves). See section 5 for realistic travel rates, population sizes, and epidemiological parameters for influenza.

If both the regional reproduction numbers are greater than 1 , increasing $\beta^{T}$ does not have a significant effect on the disease outbreak: although the curves peak earlier for larger values 


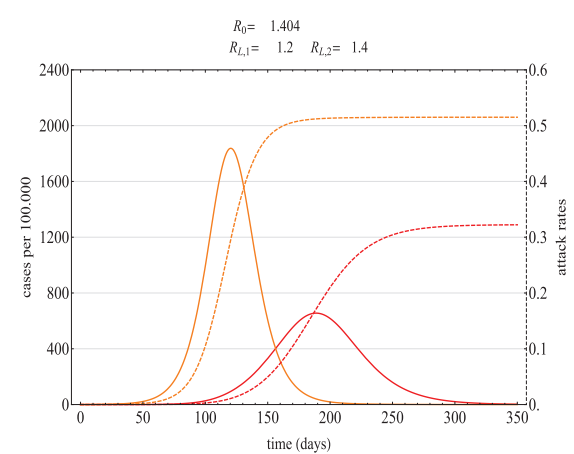

(a) Peak time in region 1: day 178; peak time in region 2: day 122 , when $\mathcal{R}_{L, 1}=1.2, \mathcal{R}_{L, 2}=1.4$, $\beta^{T}=25$.

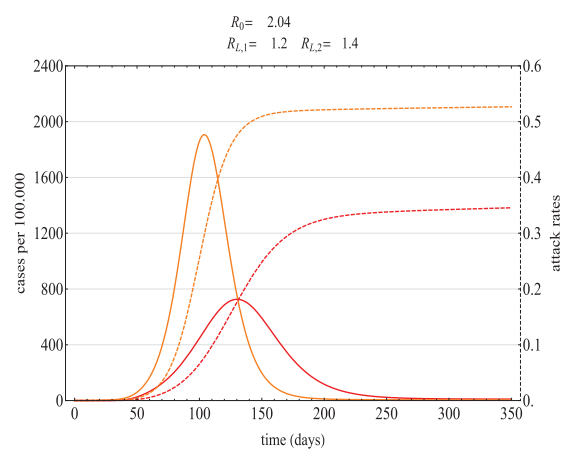

(b) Peak time in region 1: day 126; peak time in region 2: day 103, when $\mathcal{R}_{L, 1}=1.2, \mathcal{R}_{L, 2}=1.4$, $\beta^{T}=50$.

Figure 6. Epidemic curves and attack rates of region 1 (red and red-dashed) and region 2 (orange and orange-dashed) for two values of $\beta^{T}$ when both the local reproduction numbers are greater than 1 . The increase in the value of $\beta^{T}$ significantly alters the value of $\mathcal{R}_{0}$, but its effect on the epidemic curves only manifests in earlier peak times. Parameter values were chosen as $\mathcal{R}_{L, 1}=1.2, \mathcal{R}_{L, 2}=1.4, \tau=0.5, \mu_{E}^{-1}=1.4, \mu_{I}^{-1}=3$, $\mu_{A}^{-1}=4.1, p=0.6, \rho=0.1, \gamma_{1}^{-1}=\gamma_{2}^{-1}=7, \alpha_{1}=5 \cdot 10^{-5}, \alpha_{2}=5 \cdot 10^{-5}, N_{1}^{r}(0)=3.4 \cdot 10^{7}, N_{2}^{r}(0)=3.4 \cdot 10^{7}$.

of the parameter, peak sizes and attack rates (defined as the fraction of individuals who have contracted the disease) remain similar in both regions. However, as illustrated in Figure 6, the difference manifests in the value of the basic reproduction number: changing $\beta^{T}$ from 25 to 50 can increase $\mathcal{R}_{0}$ from 1.4 to 2 in this particular case.

There is no epidemic outbreak in the absence of travel if we assume that both regional reproduction numbers are less than 1 . In case of connected regions, minor outbreaks can occur if the value of $\beta^{T}$ is set to ensure that $\mathcal{R}_{0}$ exceeds 1 . However, further increasing $\beta^{T}$ may result in long-continued outbreaks with small peak sizes but relatively high values of the reproduction number and the attack rates, as indicated in Figure 7. These examples clearly show that although $\mathcal{R}_{0}$ works as a threshold regarding the stability of the disease-free state, as proved in section 4 (that is relevant to the initial growth of an epidemic), it is not necessarily a good predictor for the entire course of the outbreak and the attack rates.

5. Parametrization for influenza. We parametrize our model for the $2009 \mathrm{~A}(\mathrm{H} 1 \mathrm{~N} 1)$ pandemic influenza. We ignore demography and set parameters $\Lambda_{j}, d_{j}^{r}$, and $d_{j}^{v}, j \in\{1,2\}$, equal to 0 ; moreover, we neglect the possibility of disease induced mortality and let $\delta=0$. Several studies ([1, 4, 6, 7, 12, 20,33, 35, 36] and the references therein) have estimated the local reproduction number and values of key epidemiological parameters for recent influenza pandemics. In Table 3 we give an overview of the ranges of these parameters and choose reasonable values from the ranges for our simulations. Parameter $\beta^{T}$, the transmission rate during travel, is estimated to be 10-20, as [36] claims that the expected number of H1N1 infections caused by a single infectious case varies between 5 and 10, considering transmission during an 11-hour-long flight.

In the model description, several parameters were introduced to characterize transportation between the regions: travel rate of residents from their origin, duration of visitors' stay, duration of travel. We determine the values of these parameters for specific cases: we pick 


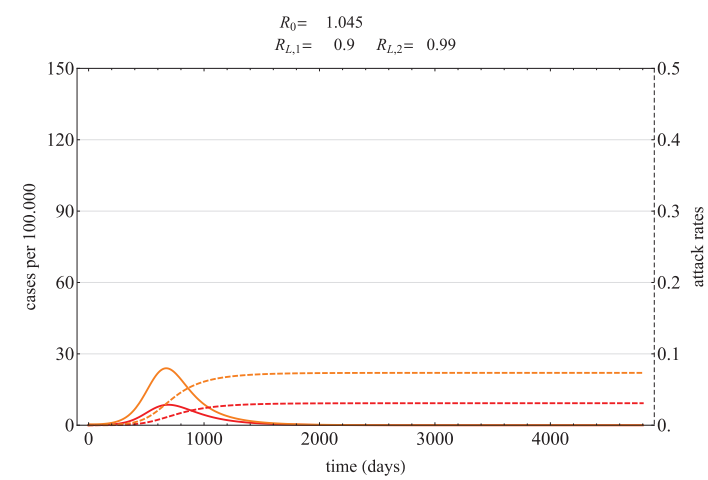

(a) $\mathcal{R}_{L, 1}=0.9, \mathcal{R}_{L, 2}=0.99, \beta^{T}=25$.

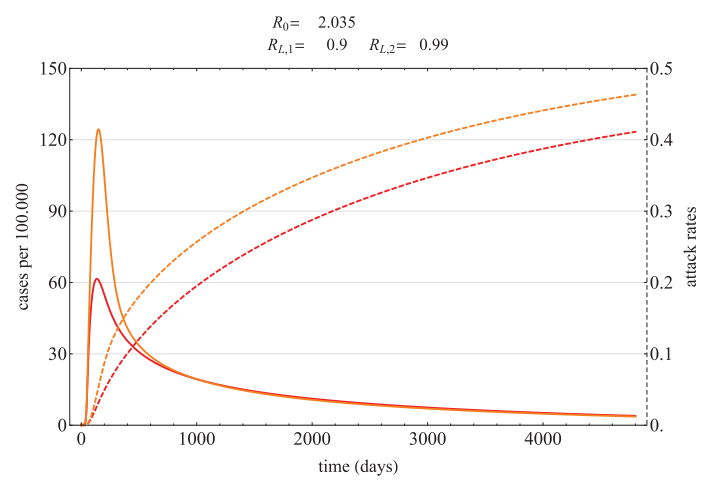

(b) $\mathcal{R}_{L, 1}=0.9, \mathcal{R}_{L, 2}=0.99, \beta^{T}=50$.

Figure 7. Epidemic curves and attack rates of region 1 (red and red-dashed) and region 2 (orange and orange-dashed) for two values of $\beta^{T}$ when both the local reproduction numbers are below 1 but $\mathcal{R}_{0}>1$. The relatively high value of the reproduction number is reflected in the size of the outbreak: the dashed curves show that for $\beta^{T}=50$ more than $40 \%$ of the populations of both regions 1 and 2 have been infected by day 4500 . Parameter values were chosen as $\mathcal{R}_{L, 1}=0.9, \mathcal{R}_{L, 2}=0.99, \tau=0.5, \mu_{E}^{-1}=1.4, \mu_{I}^{-1}=3, \mu_{A}^{-1}=4.1, p=0.6$, $\rho=0.1, \gamma_{1}^{-1}=\gamma_{2}^{-1}=7, \alpha_{1}=5 \cdot 10^{-5}, \alpha_{2}=5 \cdot 10^{-5}, N_{1}^{r}(0)=3.4 \cdot 10^{7}, N_{2}^{r}(0)=3.4 \cdot 10^{7}$.

Table 3

Parameters for simulations $(j \in\{1,2\}, m, n \in\{r, v\})$.

\begin{tabular}{|c||c|cl|}
\hline Parameter & Value for simulations & Range from literature \\
\hline$\Lambda_{j}, d_{j}^{m}, \delta$ & 0 & & \\
$\mu_{E}^{-1}$ & 1.4 & $(1-2.62)$ & {$[4,33,35]$} \\
$\mu_{I}^{-1}$ & 3 & $(2.06-4.69)$ & {$[20,33]$} \\
$\mu_{A}^{-1}$ & 4.1 & $(0-0.5)$ & {$[1,6,20]$} \\
$\rho$ & 0.1 & $(0.5-0.75)$ & {$[1,7]$} \\
$p$ & 0.6 & $(10-20)$ & {$[12,36]$} \\
$\beta^{T}$ & 15 & $(1.05-1.88)$ & {$[4,33,35]$} \\
$\mathcal{R}_{L}$ & $1.3,1.4$ & \\
$\beta_{j}^{m, n}$ & Calculated from $\mathcal{R}_{L}$ & & \\
$\alpha_{j}, \gamma_{j}, \tau$ & See specific cases & & \\
\hline
\end{tabular}

Canada to be region 1 (origin), and we consider three possible destinations as region 2 (for the destinations, see section 6). We derive travel rates from [2], which provides the annual volume of passengers toward Canada from several international sources. However, neither records about travelers' origin (Canadian resident or visitor of Canada) nor data about the volume of passengers traveling in other directions are available in this study. For many destinations available from Canada, the surveys of Statistics Canada [29] provide information about the ratio of the volume of foreign travelers to Canada and Canadian travelers to the other region. We assume that all Canadian residents who leave Canada will return some time later; therefore, for a specific region 2, the ratio of the annual volume of residents of this region traveling to Canada and Canadians returning home from the region is explicitly given by the statistics. Thus, given the annual volume of all passengers from a specific region 2 toward Canada, we can determine $\Omega_{2}$, the annual volume of residents of region 2 traveling to Canada, and the annual volume of Canadian residents returning home from region 2. We assume that this 
Table 4

Annual travel volumes and statistics for three origin-destination pairs. The annual volumes of passengers toward Canada were derived from [2]. The statistics of [29] provide the ratio of Canadian residents and residents of the other region (China/the United Kingdom/Mexico).

\begin{tabular}{|c||c|c|}
\hline Origin-destination pair & Biospora [2] & Statistics Canada 2009 [29] \\
\hline Canada - China & 786.569 & $9: 5$ \\
Canada - UK & 1.203 .272 & $14: 11$ \\
Canada - Mexico & 655.219 & $15: 2$ \\
\hline
\end{tabular}

number equals $\Omega_{1}$, the annual volume of Canadian passengers to region 2 . The annual volume of passengers toward Canada and the ratio of Canadian residents and residents of region 2 traveling between Canada and region 2 for three origin-destination pairs can be found in Table 4 .

We need $\alpha_{1}$ and $\alpha_{2}$, the traveling rates of Canadian residents and residents of region 2. In our simulations, we define the traveling rate of residents of region $j$ at time $t$ as $\alpha_{j}(t)=\frac{\Omega_{j}}{365} \frac{1}{N_{j}^{r}(t)}, j \in\{1,2\}$. (The definition is slightly different in the case of the origindestination pair Canada-Mexico; see the corresponding subsection.) Parameters $\gamma_{j}$ and $\tau$ are determined for each specific origin-destination pair. Initial values are set as follows:

$$
\begin{aligned}
& S_{j}^{r}(u)= \begin{cases}\left(1--10^{-5}\right) M_{j} & \text { if } u=0, \\
M_{j} & \text { if } u<0,\end{cases} \\
& S_{j}^{v}(u) \equiv \frac{\Omega_{k}}{365 \gamma_{j}}, \\
& E_{j}^{r}(u)= \begin{cases}10^{-5} M_{j} & \text { if } u=0, \\
0 & \text { if } u<0,\end{cases}
\end{aligned}
$$

where $M_{j}$ denotes the population size of region $j$, and $E_{j}^{v}(u)=A_{j}^{m}(u)=I_{j}^{m}(u)=R_{j}^{m}(u) \equiv 0$ for $u \in[-\tau, 0], j, k \in\{1,2\}, j \neq k, m \in\{r, v\}$. We determine the transmission rates $\beta_{j}^{m, n}$, $j \in\{1,2\}, m, n \in\{r, v\}$, as follows. For each origin-destination pair of our model, we denote the local reproduction number of region $j$ (i.e., the reproduction number of the region in the absence of travel) by $\mathcal{R}_{L, j}$. Several recent studies (e.g., [4, 33]) have estimated the basic reproduction number for regions which were affected by the 2009 H1N1 pandemic. For a given $\mathcal{R}_{L, j}$, we can use the formula

$$
\mathcal{R}_{L, j}=\beta_{j} \frac{S_{j}^{r}(0)}{N_{j}^{r}(0)}\left(\frac{p}{\mu_{I}}+(1-p) \frac{\rho}{\mu_{A}}\right)
$$

to calculate $\beta_{j}$. Assuming homogeneous mixing in the regions, we can set $\beta_{j}^{r r}=\beta_{j}^{r v}=\beta_{j}^{v r}=$ $\beta_{j}^{v v}=\beta_{j}$. For the numerical simulations we set $\mu_{E}^{T}=\mu_{A}^{T}=\mu_{I}^{T}=0$; as pointed out in section 4 , this assumption allows us to obtain the inflow terms explicitly and also to calculate the reproduction number.

As supplemental material, three animations complement this paper to facilitate the understanding of the model. Choosing reasonable values for epidemic parameters, population sizes, and travel rates, we display the epidemic curves of the two regions as time elapses. To point out the importance of distinguishing local residents from temporary visitors in the model 
setup, we also present bar charts to illustrate how the cumulative number of cases imported by residents and visitors evolves over time. At a given time $t$, the bar charts show the total number of exposed, symptomatic, and asymptomatic infected individuals who have arrived to the specific region upon completing a trip until time $t$. The lighter-colored parts of the bar charts indicate those who got infected on the plane. Although the role of different travel rates or the elevated transmission potential during travel may remain hidden by considering only the epidemic curves, looking at the cumulative number of cases imported by travelers clearly helps to understand how the infection spreads to disease-free regions. From the perspective of pandemic preparedness this issue is of particular interest. See the supplemental notes (section 8) for detailed description of the animations.

6. Prototype origin-destination pairs. In this section, we present simulations for influenza using real demographic and air traffic data. We set up three distinct scenarios for the origin-destination pairs: we choose Canada to represent region 1 and consider three possible geographic locations for region 2: China, Mexico, and the United Kingdom.These three countries are popular destinations of flights originating from Canada. We would like to emphasize that although we used real demographic and air travel data in the simulations and chose reasonable parameter values from the ranges of Table 3, the epidemic curves depicted below do not need to match with the 2009 A(H1N1) influenza pandemic data reported, since there were other factors which are not considered in our model setting. The purpose of choosing such origin-destination pairs for simulations was to illustrate our model for regions parametrized with different values of the key model parameters. Nevertheless, we also present the data fitting results of the model to the Canadian and Mexican morbidity data of the first wave of the pandemic, since in the early stage, these data were largely determined by the characteristics of the two countries and the travel within them.

Table 4 summarizes real air traffic data and the ratios of Canadian residents traveling to region 2 versus residents of region 2 traveling to Canada for three origin-destination pairs.

6.1. Canada-China: The case of asymmetric populations. China, including Hong Kong, generates the third largest volume of international passenger traffic entering Canada: approximately 780,000 air passengers initiate their trips from within China's borders. In terms of global preparedness against worldwide spreading pandemics China is of particular interest: its variability of poverty and wealth provides a platform for the appearance of emerging infectious diseases (e.g., H5N1, SARS). The country's high connectivity with all parts of the world (it possesses the fifth largest international airport in the world) clearly shows its significance in international spreading of diseases.

In the model construction we assumed that each individual of the population has equal chances to travel. This generalization is definitely not fulfilled in a population of more than 1 billion with various social-economic background; hence here we use a somewhat smaller population of potential travelers from China (150 million). The population size of Canada is set to 34.461 million; we let $\tau=0.5$ (days), since we assume that a flight between the regions takes approximately 12 hours. For the local reproduction numbers of Canada and China, we pick $\mathcal{R}_{L, 1}=1.3$ and $\mathcal{R}_{L, 2}=1.4$. Parameters $\alpha_{1}$ and $\alpha_{2}$ were derived using travel volumes as explained in section 5 , and we choose $\gamma_{1}=\gamma_{2}=\frac{1}{7}$.

We present Figures 8 and 9 to demonstrate the role of the human transportation system 


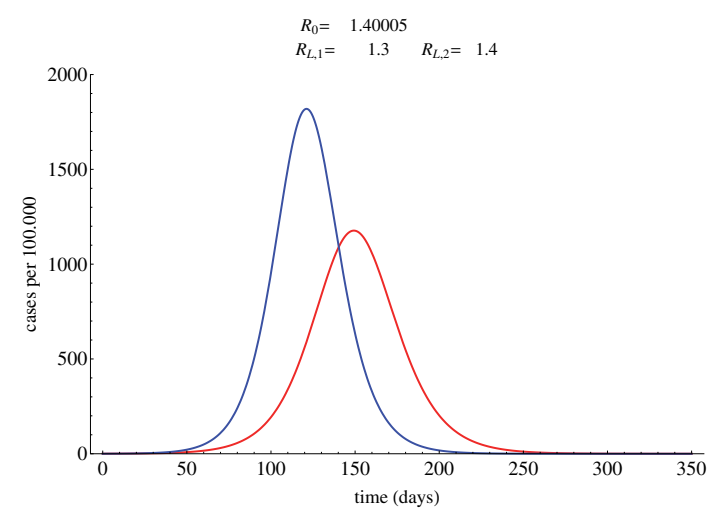

(a) Peak time in Canada: day 149; peak time in China: day 125 in the case of regions connected by air travel, independent outbreaks.

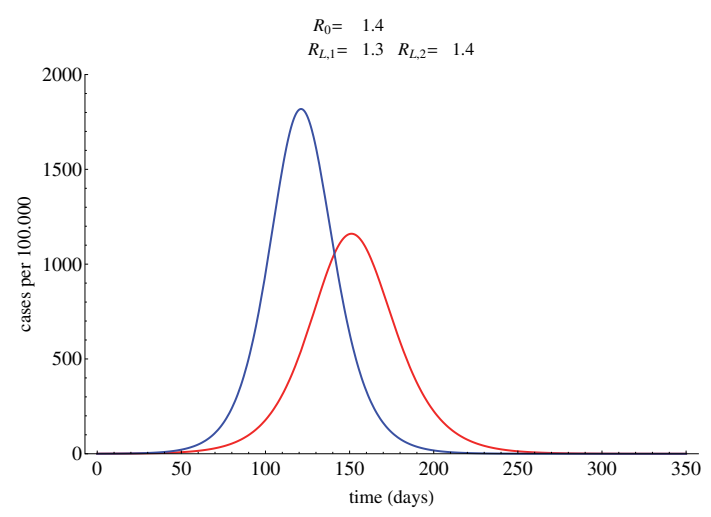

(b) Peak time in Canada: day 152; peak time in China: day 125 in the case of separated regions, independent outbreaks.

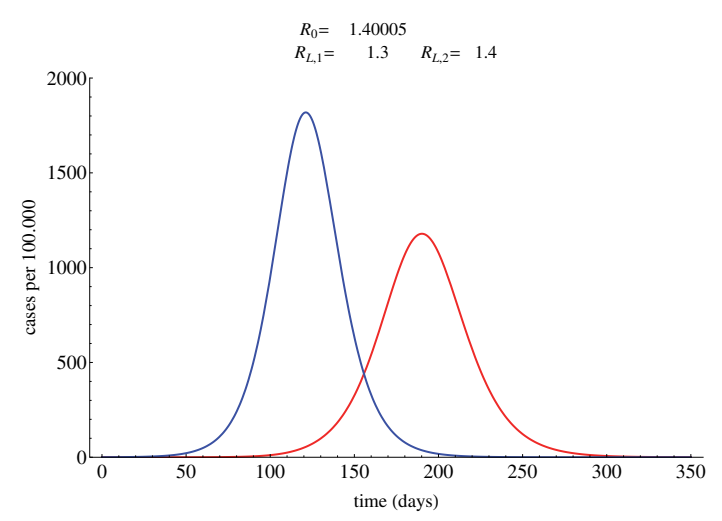

(c) Peak time in Canada: day 190; peak time in China: day 125 in the case of regions connected by air travel, initial cases only in China.

Figure 8. Epidemic curves of Canada (region 1, red) and China (region 2, blue). In case of independent outbreaks in Canada and China, the no effect of travel on the spread of the pandemic is apparent. However, if we assume that the pandemic originates from China and that Canada is susceptible, then due to air transportation the infection invades the disease-free Canada, though with delayed peak time. For the simulations, we set $\mathcal{R}_{L, 1}=1.3, \mathcal{R}_{L, 2}=1.4, \tau=0.5, \gamma_{1}^{-1}=\gamma_{2}^{-1}=7, \beta^{T}=15, \mu_{E}^{-1}=1.4, \mu_{I}^{-1}=3, \mu_{A}^{-1}=4.1, p=0.6, \rho=0.1$.

in the spread of influenza. Assuming that initial outbreaks in Canada (red curves) and China (blue curves) occurred independently, the effect of traveling (Figure 8(b)) on the spread of the epidemic appears much smaller than in the case of separated regions (Figure 8(a)). However, Figure 8(c) clearly shows the importance of incorporating transportation into the model: if we assume that initial cases appear only in China and that Canada is completely susceptible, then the disease reaches Canada, obviously due to air transportation. This scenario results in approximately the same peak size as if we assumed initial outbreaks in both regions, although the peak time in Canada is delayed by almost 40 days.

Analyzing the public reports of health agencies confirms that large delays between peak times of connected regions are unrealistic when one considers pandemics, where air trans- 


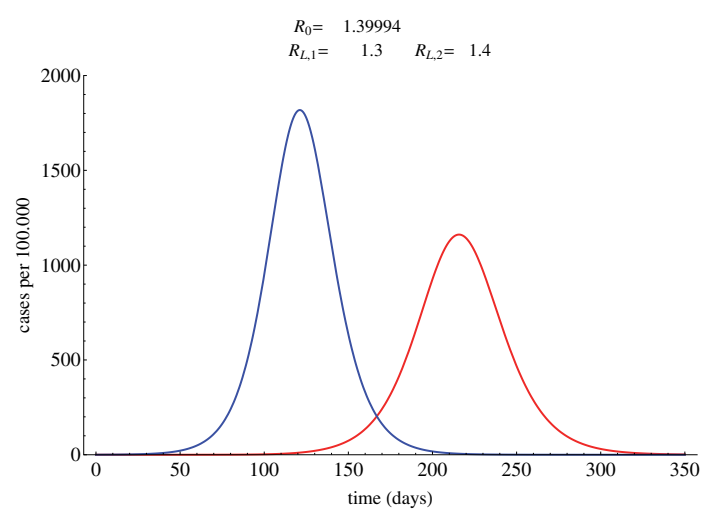

Figure 9. Epidemic curves of Canada (region 1, red) and China (region 2, blue) when we ignore the possibility of disease transmission during travel and we assume that the pandemic originates from China and that Canada is susceptible. Peak time in Canada: day 220, peak time in China: day 125. For the simulations, we set $\mathcal{R}_{L, 1}=1.3, \mathcal{R}_{L, 2}=1.4, \tau=0.5, \gamma_{1}^{-1}=\gamma_{2}^{-1}=7, \beta^{T}=15, \mu_{E}^{-1}=1.4, \mu_{I}^{-1}=3, \mu_{A}^{-1}=4.1, p=0.6$, $\rho=0.1$.

portation was proved to play a key role in disease transmission (SARS in 2002-2003 and $\mathrm{A}(\mathrm{H} 1 \mathrm{N1}) \mathrm{v}$ influenza in 2009). Figure 9 shows what happens if we ignore the possibility of on-board disease transmission in the model. Comparing this result with Figure 8(c) is of particular interest: if one incorporates disease dynamics during travel, the model predicts the peak time of the invaded region to be 30 days earlier. As a concluding remark, we wish to emphasize that, following these findings, simpler models that ignore travel-related infections can seriously overestimate the time a region has for preparation before the outbreak arrives.

6.2. Canada-United Kingdom: The symmetric case. The European Union (EU) generates almost one-fifth of all international traffic entering Canada. Although the EU may be an unlikely source for the emergence of new or dangerous infectious disease threats, it generates over $19 \%$ of the world's international traffic volume and consequently should receive special consideration as an important potential location from which infectious disease threats may enter Canada. Being the second leading international source of passenger traffic entering Canada $(6.6 \%)$, the United Kingdom is an important international traffic intersection.

Unlike the Canada-China and the Canada-Mexico origin-destination pairs, the CanadaUnited Kingdom pair can be considered as the case of two symmetric regions. Their population sizes have the same magnitude; moreover, just like Canada, the UK also possesses a highly developed health care system and advanced intervention techniques in disease control and prevention, and hence we can assume that the local reproduction numbers in the two countries are similar. In the simulations we set the population size of the UK to be 62.262 million, $\mathcal{R}_{L, 1}=\mathcal{R}_{L, 2}=1.3, \gamma_{1}=\gamma_{2}=\frac{1}{7}$, and $\tau=0.5$.

Reducing the number of flights to and from infected areas and screening out infected individuals at their arrival to international airports are considered to be powerful tools when one's aim is to mitigate the severity of pandemic outbreaks. However, entry screening works ineffectively an asymptomatic infected individuals, who - despite their reduced disease transmissibility can spread the infection after arrival. We examined the benefits of travel restrictions on the 


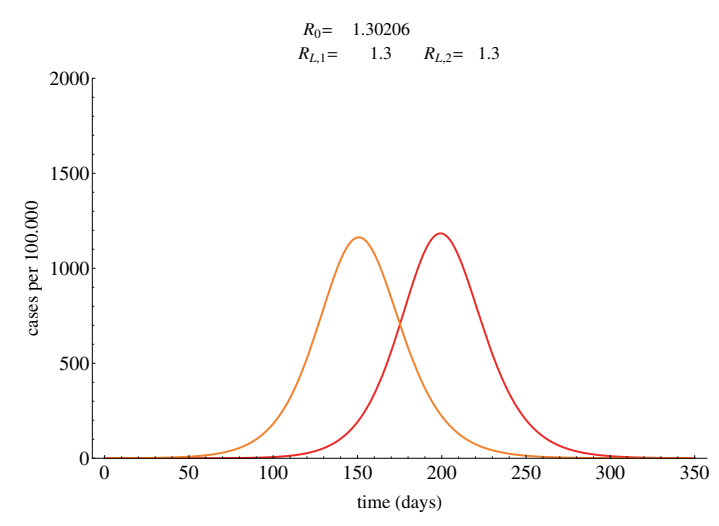

(a) Peak time in Canada: day 200; peak time in the UK: day 153 when there are no reduction in travel volumes.

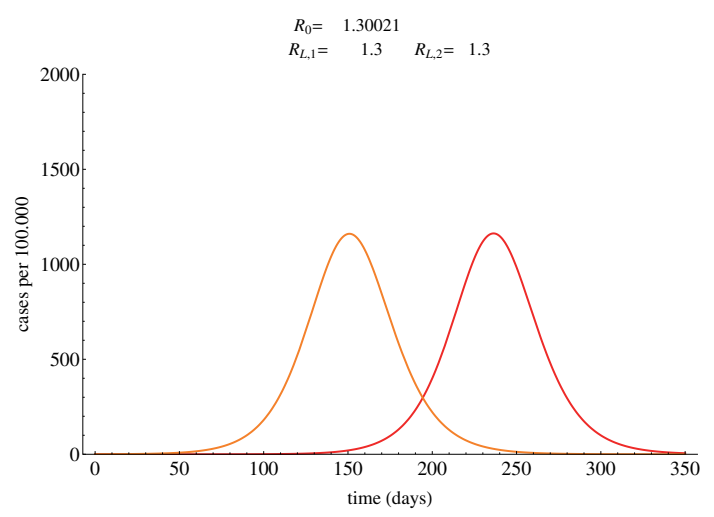

(b) Peak time in Canada: day 240; peak time in the UK: day 153 when there is a $90 \%$ reduction in travel volumes.

Figure 10. Reducing travel volumes by $90 \%$ between Canada (region 1, red) and the UK (region 2, orange) results in a delay of the peak time in Canada by 40 days. We assumed the initial number of infectious cases in Canada to be 0. For the simulations, we set $\mathcal{R}_{L, 1}=1.3, \mathcal{R}_{L, 2}=1.3, \tau=0.5, \gamma_{1}^{-1}=\gamma_{2}^{-1}=7, \beta^{T}=15$, $\mu_{E}^{-1}=1.4, \mu_{I}^{-1}=3, \mu_{A}^{-1}=4.1, p=0.6, \rho=0.1$.

spread of the pandemic in two symmetric regions. We found that in the case of a single outbreak in the UK, the peak time of the originally disease-free Canada outbreak can be delayed by 40 days when we consider the hypothetical case of $90 \%$ limitation in the travel volumes between the two regions. See Figure 10: red and orange curves show the number of symptomatic infected cases per 1,000,000 residents in Canada and the UK, respectively.

6.3. Canada-Mexico: The case of asymmetric travel. Mexico is a potential source of threatening pandemics due to high population density, poverty, and limited health care resources on one side but high volume of international traffic on the other side. The country generates the forth largest volume of international passenger traffic entering Canada (approximately 655,000 passengers, second largest volume from any developing countries). A significantly large part of this traffic originates from resort cities like Cancun $(177,000)$ and Puerto Vallarta $(105,000)$ between January and April, as Canadians return home from winter vacations. However, Mexico City is a steady source of inflowing air traffic throughout the year $(190,000)$. This asymmetric travel behavior may cause very dissimilar epidemic courses if we consider outbreaks in different parts of the year.

In order to incorporate the phenomenon of this asymmetric travel behavior of passengers traveling between Canada and Mexico, we divide the course of the year into two phases. Between January and April (Phase I) the daily volume of Canadian residents traveling to Mexico is significantly higher than during the other 8 months of the year (May-December, Phase II). We denote the travel rates of Canadian residents toward Mexico in Phases I and II by $\alpha_{1}^{I}$ and $\alpha_{1}^{I I}$, respectively. Parameter $\Omega_{1}$ was introduced in section 5 to denote the annual volume of Canadians entering Mexico, and using the data of Table 4, we obtain that $\Omega_{1}=655000 \frac{15}{15+2}$. We define $\alpha_{1}(t)$, the traveling rate of Canadian residents toward Mexico at time $t$, as follows: we assume that the passenger traffic from Canada to resort cities like 


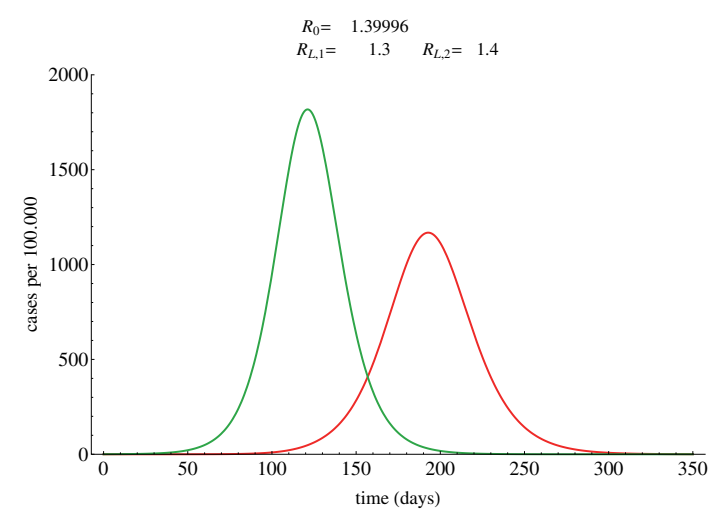

(a) Peak time in Canada: day 195; peak time in Mexico: day 123 when $\gamma_{1}=\frac{1}{15}, \gamma_{2}=\frac{1}{15}$.

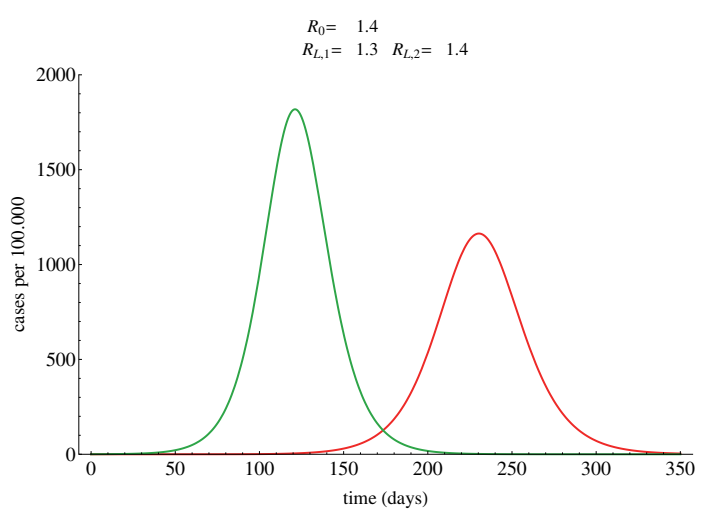

(b) Peak time in Canada: day 230; peak time in Mexico: day 123 when $\gamma_{1}=\frac{1}{0.2}, \gamma_{2}=\frac{1}{0.2}$.

Figure 11. In case of a single initial outbreak in Mexico (region 2, green), the outbreak in Canada (region 1 , red) can be delayed by 35 days if the average stay of visitors is reduced from 15 days to 5 hours. For the simulations, we set $\mathcal{R}_{L, 1}=1.3, \mathcal{R}_{L, 2}=1.4, \tau=0.25, \beta^{T}=15, \mu_{E}^{-1}=1.4, \mu_{I}^{-1}=3, \mu_{A}^{-1}=4.1, p=0.6$, $\rho=0.1$.

Cancun and Puerto Vallarta is due to Canadian residents only, who travel to Mexico for vacation, and all travelers to these cities arrive to Mexico during the first four months of the year. We denote the number of all residents of region 1 (Canada) at time $t$ by $N_{1}^{r}(t)$. We get that

$$
\alpha_{1}(t)=\alpha_{1}^{I I}=\frac{\Omega_{1}-177000-105000}{365} \frac{1}{N_{1}^{r}(t)}
$$

for $t>121$, i.e., in Phase II, and

$$
\alpha_{1}(t)=\alpha_{1}^{I}=\alpha_{1}^{I I}+\frac{177000+105000}{121} \frac{1}{N_{1}^{r}(t)}
$$

for $0 \leq t \leq 121$, where $t=0$ corresponds to December 31, 2008 .

As mentioned above, a significant part of the passenger traffic between Canada and Mexico is due to Canadian vacationers who visit holiday resorts in the first four months of the year. Although the news about an epidemic outbreak might not make people cancel their vacation, it might affect the length of their stay in the affected area. We considered two scenarios for the average length of visitors' stay to reveal the importance of this time period. Figure 11 shows that the smaller the value of parameters $\frac{1}{\gamma_{1}}$ and $\frac{1}{\gamma_{2}}$ is, the later the pandemic hits Canada (red curve) if we assume that the first cases were identified in Mexico (green curve). If visitors spend 15 days on average in the other region, the pandemic peaks 35 days earlier in Canada than if we consider a 0.2 day-long (approximately 5 hours, usual waiting time of transit passengers at airports) stay only. These results were obtained using parameter values $\tau=0.25, \mathcal{R}_{L, 1}=1.3, \mathcal{R}_{L, 2}=1.4$, and the population size of Mexico was set to 112.323 million.

6.4. Fitting the model to the $2009 \mathrm{~A}(\mathrm{H} 1 \mathrm{~N} 1)$ pandemic. To illustrate the applicability of our approach, we fitted the model to the first wave of the $2009 \mathrm{~A}(\mathrm{H} 1 \mathrm{~N} 1) \mathrm{v}$ pandemic 


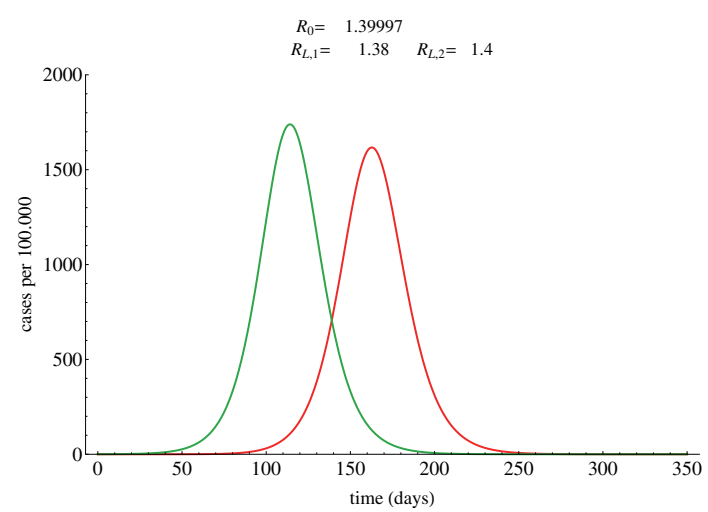

Figure 12. Epidemic curves of Canada (region 1, red, peak time: day 160) and Mexico (region 2, green, peak time: 117) when peak times were fitted to the real morbidity data of the first wave of the 2009 H1N1 outbreak and day 0 corresponds to December 31, 2008. Travel rates arise from [2], and historical peak times (day 117-123 in Mexico, day 155-162 in Canada) were derived from [21, 24, 39]. We set $\mathcal{R}_{L, 1}=1.38, \mathcal{R}_{L, 2}=1.4, \tau=0.25$, $\gamma_{1}^{-1}=\gamma_{2}^{-1}=15, \beta^{T}=20, \mu_{E}^{-1}=1.4, \mu_{I}^{-1}=2.7, \mu_{A}^{-1}=4.1, p=0.6, \rho=0.1$.

in Canada and Mexico. For the simulation, we chose reasonable values for epidemiological parameters from the ranges of Table 3; moreover, we used real demographic and air traffic data of the Canada-Mexico origin-destination pair. Travel rates were derived from [2], and we set $\tau=0.25$. According to the public reports of the Mexican Social Security Institute [21], WHO Global Influenza Virological Surveillance [39], and the Public Health Agency of Canada [24], the epidemic peaked around week 18 in Mexico and week 23-24 in Canada. If day 0 corresponds to December 31, 2008, then historical peak times are obtained around day 117-123 in Mexico and around day 155-162 in Canada. For the simulations we estimated the local reproduction numbers to ensure that the peak times of the epidemic curves fit the real morbidity data. The result can be seen in Figure 12, where $\mathcal{R}_{L, 1}=1.38$ and $\mathcal{R}_{L, 2}=1.4$. These local reproduction numbers match the results of $[4,33,35]$.

We performed a systematic analysis to reveal the sensitivity to several key parameters. The analysis showed the robustness of the presented fitting in parameters $\gamma_{1}, \gamma_{2}, p$, and $\rho$, although it turned out that the length of latency and infectious periods, the transmission rate during travel, and the local reproduction numbers strongly affect the peak times. We wish to emphasize the utmost importance of incorporating disease dynamics during transportation into our model. The discussion around Figures 8(c) and 9 in section 6 clearly shows that ignoring the possibility of on-board disease transmission results in delayed peak times; thus by ignoring travel related infections while keeping every other parameter fixed, we could not have had a fitting as presented in Figure 12. To ensure that the curve of Canada peaks around day 155-162 in the absence of travel infections, a much higher value for the Canadian reproduction number $\mathcal{R}_{L, 1}$ would be necessary, which is unrealistic according to the abovementioned references.

7. Conclusion. Recent epidemics like the 2002-2003 SARS outbreak and the 2009 pandemic influenza $\mathrm{A}(\mathrm{H} 1 \mathrm{~N} 1)$ demonstrated the role that the global air transportation network played in the worldwide spread of infectious diseases. The topic of epidemic spread due to hu- 
man transportation has recently been examined in several studies. The meta-population models in $[2,3,25,37]$ describe the spatial dispersal of infected individuals in connected regions, although ignoring the fact that long-distance travel such as intercontinental flights provides a platform for on-board transmission of the disease [12, 36]. The studies [8, 31, 18, 22, 23] account for the fact that since the progress of the above-mentioned diseases is fast, the time needed for transportation between regions is not negligible. They consider the possibility of disease transmission during travel, although the standard SIS-type models used in these studies might not be suitable for modeling influenza or SARS.

We introduced a dynamic model which describes the spread of an infectious disease within and between two regions which are connected by transportation. In the model setup we distinguished local residents from temporary visitors because they might have very different contact rates, mixing patterns, and travel behavior. We used the SEAIR model as a basic epidemic building block in the regions and also during travel, and we modeled disease dynamics during travel by a system structured by travel time. We showed that our model is equivalent to a system of nonlinear functional differential equations with dynamically defined delayed feedback, and we examined the fundamental dynamic properties of the system.

We detailed the computation of the basic reproduction number, which is a threshold quantity for epidemic outbreaks, and discussed the dependence of $\mathcal{R}_{0}$ on several key model parameters. The analysis demonstrates the importance of incorporating the phenomenon of disease transmission during transportation: transmission rates during travel can be much higher than under usual circumstances, and our results show that $\beta^{T}$ may significantly alter the value of $\mathcal{R}_{0}$. We parametrized our model for influenza and performed simulations with real demographic and air traffic data. Three origin-destination pairs were introduced for the regions to demonstrate the effect of changing the value of various key model parameters and addressing possible interventions. We showed the applicability of our approach by fitting the model to the first wave of the 2009 A(H1N1) influenza pandemic in Canada and Mexico.

Our results, in conjunction with recent studies $[12,18,36]$, support that considering disease transmission during travel is of particular interest to modeling the spread of diseases with fast progression. We demonstrated that simpler models which ignore on-board infections can seriously overestimate the time a region has before the epidemic wave arrives.

8. Supplemental notes. The following animations are provided online.

91412_01.mov [local/web 484KB]: Epidemic curves of region 1 (red) and region 2 (green) as time (in days) evolves. The bar charts show the cumulative number of cases (exposed, symptomatic and asymptomatic infected residents/visitors) imported to the regions by means of travel. The lighter-colored parts of the bar charts indicate those who got infected during travel. We consider the case when the two regions are symmetric in the population sizes, travel rates, and values of every model parameter except the local reproduction numbers. Parameter values were chosen as $\mathcal{R}_{L, 1}=1.2, \mathcal{R}_{L, 2}=1.4, \beta^{T}=25, \tau=0.5, \mu_{E}^{-1}=1.4$, $\mu_{I}^{-1}=3, \mu_{A}^{-1}=4.1, p=0.6, \rho=0.1, \gamma_{1}^{-1}=\gamma_{2}^{-1}=7, \alpha_{1}=5 \cdot 10^{-5}, \alpha_{2}=5 \cdot 10^{-5}$, $N_{1}^{r}(0)=3.4 \cdot 10^{7}, N_{2}^{r}(0)=3.4 \cdot 10^{7}$.

91412_02.mov [local/web 465KB]: Epidemic curves of region 1 (red) and region 2 (blue) as time (in days) evolves. The bar charts show the cumulative number of cases (exposed, symptomatic and asymptomatic infected residents/visitors) imported to the regions by means 
of travel. The lighter-colored parts of the bar charts indicate those who got infected during travel. We consider the case when the two regions are symmetric in the population sizes and values of every model parameter, but have different local reproduction numbers, and we assume that the travel rate of residents in region 2 is four times the travel rate of residents in region 1. Although the effect of this asymmetry does not seem to manifest on the epidemic curves in comparison to the previous case, the bar charts clearly show the difference in the ratio of cases imported to region 1 by residents and visitors. Parameter values were chosen as $\mathcal{R}_{L, 1}=1.2, \mathcal{R}_{L, 2}=1.4, \beta^{T}=25, \tau=0.5, \mu_{E}^{-1}=1.4, \mu_{I}^{-1}=3, \mu_{A}^{-1}=4.1, p=0.6, \rho=0.1$, $\gamma_{1}^{-1}=\gamma_{2}^{-1}=7, \alpha_{1}=2 \cdot 10^{-5}, \alpha_{2}=8 \cdot 10^{-5}, N_{1}^{r}(0)=3.4 \cdot 10^{7}, N_{2}^{r}(0)=3.4 \cdot 10^{7}$.

91412_03.mov [local/web 492KB]: Epidemic curves of region 1 (red) and region 2 (orange) as time (in days) evolves. The bar charts show the cumulative number of cases (exposed, symptomatic and asymptomatic infected residents/visitors) imported to the regions by means of travel. The lighter-colored parts of the bar charts indicate those who got infected during travel. We consider the case when the two regions are symmetric in the population sizes, travel rates, and values of every model parameter except the local reproduction numbers. For this simulation, we use a relatively high value for the transmission rate during travel to demonstrate its effect on the number of imported cases. Parameter values were chosen as $\mathcal{R}_{L, 1}=1.2, \mathcal{R}_{L, 2}=1.4, \beta^{T}=50, \tau=0.5, \mu_{E}^{-1}=1.4, \mu_{I}^{-1}=3, \mu_{A}^{-1}=4.1, p=0.6, \rho=0.1$, $\gamma_{1}^{-1}=\gamma_{2}^{-1}=7, \alpha_{1}=5 \cdot 10^{-5}, \alpha_{2}=5 \cdot 10^{-5}, N_{1}^{r}(0)=3.4 \cdot 10^{7}, N_{2}^{r}(0)=3.4 \cdot 10^{7}$.

Appendix. The NGM $\mathcal{N}$ for system $(\bar{L})$ was introduced in section 4 by dividing all exposed individuals into four groups according to their region and residential status. The elements can be obtained by following the way of infection considering our definition of reproduction. Here we detail the calculation of two elements of $\mathcal{N}$ and then show that they equal the corresponding elements of $\mathcal{K}$, the NGM of the associated system of ODEs (15). Formulas for other elements - and hence the equalities $(\mathcal{N})_{j, k}=(\mathcal{K})_{j, k}, j, k \in\{1,2,3,4\}$-can be derived similarly.

First, let us consider the element $R_{11}^{r r}$, namely the number of new infections in $E_{1}^{r}$ generated by an exposed resident of region 1 (a member of $E_{1}^{r}$ ). Since in the model setup we addressed no restrictions on the number of travels an individual can start, we distinguish two scenarios: (A) After completing some even number of travels, the exposed individual turns infected (member of class $I$ or $A$ ) in region 1 .

(B) After completing some odd number of travels, the exposed individual turns infected in region 2 .

We calculate the probabilities of these events. First, the probability of turning infected after $(2 n)$ times of travel, $n=0,1,2 \ldots$, arises as

$$
\left(\frac{\alpha_{1}}{\alpha_{1}+\mu_{E}+d_{1}^{r}} \frac{\gamma_{2}}{\gamma_{2}+\mu_{E}+d_{2}^{v}}\right)^{n} \frac{\mu_{E}}{\alpha_{1}+\mu_{E}+d_{1}^{r}},
$$

which implies that

$$
\begin{aligned}
P(A) & =\sum_{n=0}^{\infty}\left(\frac{\alpha_{1}}{\alpha_{1}+\mu_{E}+d_{1}^{r}} \frac{\gamma_{2}}{\gamma_{2}+\mu_{E}+d_{2}^{v}}\right)^{n} \frac{\mu_{E}}{\alpha_{1}+\mu_{E}+d_{1}^{r}} \\
& =\frac{\mu_{E}}{\alpha_{1}+\mu_{E}+d_{1}^{r}} \sum_{n=0}^{\infty}\left(\frac{\alpha_{1}}{\alpha_{1}+\mu_{E}+d_{1}^{r}} \frac{\gamma_{2}}{\gamma_{2}+\mu_{E}+d_{2}^{v}}\right)^{n}
\end{aligned}
$$




$$
\begin{gathered}
=\frac{\mu_{E}}{\alpha_{1}+\mu_{E}+d_{1}^{r}} \cdot \frac{1}{1-\frac{\alpha_{1}}{\alpha_{1}+\mu_{E}+d_{1}^{r}} \frac{\gamma_{2}}{\gamma_{2}+\mu_{E}+d_{2}^{v}}} \\
=\frac{\mu_{E}}{\alpha_{1}+\mu_{E}+d_{1}^{r}} \cdot \frac{\left(\alpha_{1}+\mu_{E}+d_{1}^{r}\right)\left(\gamma_{2}+\mu_{E}+d_{2}^{v}\right)}{\left(\alpha_{1}+\mu_{E}+d_{1}^{r}\right)\left(\gamma_{2}+\mu_{E}+d_{2}^{v}\right)-\alpha_{1} \gamma_{2}} \\
=\frac{\mu_{E}\left(\gamma_{2}+\mu_{E}+d_{2}^{v}\right)}{\left(\alpha_{1}+\mu_{E}+d_{1}^{r}\right)\left(\gamma_{2}+\mu_{E}+d_{2}^{v}\right)-\alpha_{1} \gamma_{2}} .
\end{gathered}
$$

On the other hand, the probability of becoming infected after $(2 n+1)$ times of travel, $n=$ $0,1,2 \ldots$, is

$$
\left(\frac{\alpha_{1}}{\alpha_{1}+\mu_{E}+d_{1}^{r}}\right)^{n+1}\left(\frac{\gamma_{2}}{\gamma_{2}+\mu_{E}+d_{2}^{v}}\right)^{n} \frac{\mu_{E}}{\mu_{E}+\gamma_{2}+d_{2}^{v}}
$$

hence we get the probability of case (B) by the calculations

$$
\begin{aligned}
P(B) & =\sum_{n=0}^{\infty} \frac{\mu_{E}}{\mu_{E}+\gamma_{2}+d_{2}^{v}} \frac{\alpha_{1}}{\alpha_{1}+\mu_{E}+d_{1}^{r}} \cdot\left(\frac{\alpha_{1}}{\alpha_{1}+\mu_{E}+d_{1}^{r}} \frac{\gamma_{2}}{\gamma_{2}+\mu_{E}+d_{2}^{v}}\right)^{n} \\
& =\frac{\mu_{E}}{\mu_{E}+\gamma_{2}+d_{2}^{v}} \cdot \frac{\alpha_{1}}{\alpha_{1}+\mu_{E}+d_{1}^{r}} \sum_{n=0}^{\infty}\left(\frac{\alpha_{1}}{\alpha_{1}+\mu_{E}+d_{1}^{r}} \frac{\gamma_{2}}{\gamma_{2}+\mu_{E}+d_{2}^{v}}\right)^{n} \\
& =\frac{\mu_{E}}{\mu_{E}+\gamma_{2}+d_{2}^{v}} \cdot \frac{\alpha_{1}}{\alpha_{1}+\mu_{E}+d_{1}^{r}} \cdot \frac{1}{1-\frac{\alpha_{1}}{\alpha_{1}+\mu_{E}+d_{1}^{r}} \frac{\gamma_{2}+\mu_{E}+d_{2}^{v}}{\gamma_{2}}} \cdot \frac{\left(\alpha_{1}+\mu_{E}+d_{1}^{r}\right)\left(\gamma_{2}+\mu_{E}+d_{2}^{v}\right)}{\left(\alpha_{1}+\mu_{E}+d_{1}^{r}\right)\left(\gamma_{2}+\mu_{E}+d_{2}^{v}\right)-\alpha_{1} \gamma_{2}} \\
& =\frac{\mu_{E}}{\mu_{E}+\gamma_{2}+d_{2}^{v}} \cdot \frac{\alpha_{1}+\mu_{E}+d_{1}^{r}}{\alpha_{1}} \\
& =\frac{\left.\alpha_{1}+\mu_{E}+d_{1}^{r}\right)\left(\gamma_{2}+\mu_{E}+d_{2}^{v}\right)-\alpha_{1} \gamma_{2}}{\left(\alpha_{1}\right.}
\end{aligned}
$$

In case (A), an infected individual can transmit the disease in two ways:

(a1) as a member of class $I_{1}^{r}\left(A_{1}^{r}\right)$; i.e., the individual infects in region 1 after an even number of completed travels (counted since the individual became infected), or

(a2) after an odd number of completed travels (counted since the individual became infected), the individual is a member of class $I_{2}^{v}\left(A_{2}^{v}\right)$; the individual leaves region 2 and infects during travel from region 2 to region 1.

Similarly, in case (B), the two ways of disease transmission are as follows:

(b1) as a member of class $I_{1}^{r}\left(A_{1}^{r}\right)$; i.e., the individual infects in region 1 after an odd number of completed travels (counted since the individual became infected), or

(b2) after an even number of completed travels (counted since the individual became infected), the individual is a member of class $I_{2}^{v}\left(A_{2}^{v}\right)$; the individual leaves region 2 and infects during travel from region 2 to region 1.

In case (a1), the expected duration of infection of an individual in class $I_{1}^{r}$ after the $(2 n)$ th travel $(n=0,1, \ldots)$ arises as

$$
\left(\frac{\alpha_{1}}{\alpha_{1}+\mu_{I}+\delta+d_{1}^{r}} \frac{\gamma_{2}}{\gamma_{2}+\mu_{I}+\delta+d_{2}^{v}}\right)^{n} \frac{1}{\alpha_{1}+\mu_{I}+\delta+d_{1}^{r}}
$$


and we derive the total expected infection time in region 1 as

$$
\begin{aligned}
& \sum_{n=0}^{\infty}\left(\frac{\alpha_{1}}{\alpha_{1}+\mu_{I}+\delta+d_{1}^{r}} \frac{\gamma_{2}}{\gamma_{2}+\mu_{I}+\delta+d_{2}^{v}}\right)^{n} \frac{1}{\alpha_{1}+\mu_{I}+\delta+d_{1}^{r}} \\
= & \frac{1}{\alpha_{1}+\mu_{I}+\delta+d_{1}^{r}} \sum_{n=0}^{\infty}\left(\frac{\alpha_{1}}{\alpha_{1}+\mu_{I}+\delta+d_{1}^{r}} \frac{\gamma_{2}}{\gamma_{2}+\mu_{I}+\delta+d_{2}^{v}}\right)^{n} \\
= & \frac{1}{\alpha_{1}+\mu_{I}+\delta+d_{1}^{r}} \cdot \frac{1}{1-\frac{\alpha_{1}}{\alpha_{1}+\mu_{I}+\delta+d_{1}^{r}} \frac{\gamma_{2}}{\gamma_{2}+\mu_{I}+\delta+d_{2}^{v}}} \\
= & \frac{1}{\alpha_{1}+\mu_{I}+\delta+d_{1}^{r}} \cdot \frac{\left(\alpha_{1}+\mu_{I}+\delta+d_{1}^{r}\right)\left(\gamma_{2}+\mu_{I}+\delta+d_{1}^{r}\right)}{\left(\alpha_{1}+\mu_{I}+\delta+d_{1}^{r}\right)\left(\gamma_{2}+\mu_{I}+\delta+d_{2}^{v}\right)-\alpha_{1} \gamma_{2}} \\
= & \frac{\gamma_{2}+\mu_{I}+\delta+d_{2}^{v}}{\left(\alpha_{1}+\mu_{I}+\delta+d_{1}^{r}\right)\left(\gamma_{2}+\mu_{I}+\delta+d_{2}^{v}\right)-\alpha_{1} \gamma_{2}} .
\end{aligned}
$$

A similar formula holds for individuals in class $A_{1}^{r}$, so we obtain the number of new infections in case (a1) as

$$
\begin{aligned}
p & \left(\frac{\mu_{E}\left(\gamma_{2}+\mu_{E}+d_{2}^{v}\right)}{\left(\alpha_{1}+\mu_{E}+d_{1}^{r}\right)\left(\gamma_{2}+\mu_{E}+d_{2}^{v}\right)-\alpha_{1} \gamma_{2}}\right. \\
& \left.\cdot \frac{\gamma_{2}+\mu_{I}+\delta+d_{2}^{v}}{\left(\alpha_{1}+\mu_{I}+\delta+d_{1}^{r}\right)\left(\gamma_{2}+\mu_{I}+\delta+d_{2}^{v}\right)-\alpha_{1} \gamma_{2}} \beta_{1}^{r r} \frac{\hat{N}_{1}^{r}}{\hat{N}_{1}^{r}+\hat{N}_{1}^{v}}\right) \\
+(1-p) & \left(\frac{\mu_{E}\left(\gamma_{2}+\mu_{E}+d_{2}^{v}\right)}{\left(\alpha_{1}+\mu_{E}+d_{1}^{r}\right)\left(\gamma_{2}+\mu_{E}+d_{2}^{v}\right)-\alpha_{1} \gamma_{2}}\right. \\
& \left.\cdot \frac{\gamma_{2}+\mu_{A}+d_{2}^{v}}{\left(\alpha_{1}+\mu_{A}+d_{1}^{r}\right)\left(\gamma_{2}+\mu_{A}+d_{2}^{v}\right)-\alpha_{1} \gamma_{2}} \rho \beta_{1}^{r r} \frac{\hat{N}_{1}^{r}}{\hat{N}_{1}^{r}+\hat{N}_{1}^{v}}\right) .
\end{aligned}
$$

We derive the number of new infections in case (a2) similarly. Since the probability of being (symptomatic) infected just before the $(2 n+2)$ th travel $(n=0,1, \ldots)$ is

$$
\frac{\alpha_{1}}{\alpha_{1}+\mu_{I}}\left(\frac{\alpha_{1}}{\alpha_{1}+\mu_{I}+\delta+d_{1}^{r}} \frac{\gamma_{2}}{\gamma_{2}+\mu_{I}+\delta+d_{2}^{v}}\right)^{n} \frac{\gamma_{2}}{\gamma_{2}+\mu_{I}+\delta+d_{2}^{v}}
$$

and the duration of infection during the $(2 n+2)$ th travel is $\tau$, we get the total expected infection time of an individual of $I_{1}^{v}$ as

$$
\begin{aligned}
& \sum_{n=0}^{\infty} \tau\left(\frac{\alpha_{1}}{\alpha_{1}+\mu_{I}+\delta+d_{1}^{r}} \frac{\gamma_{2}}{\gamma_{2}+\mu_{I}+\delta+d_{2}^{v}}\right)^{n+1} \\
& =\tau \frac{\alpha_{1}}{\alpha_{1}+\mu_{I}+\delta+d_{1}^{r}} \cdot \frac{\gamma_{2}}{\gamma_{2}+\mu_{I}+\delta+d_{2}^{v}} \cdot \frac{1}{1-\frac{\alpha_{1}}{\alpha_{1}+\mu_{I}+\delta+d_{1}^{r}} \frac{\gamma_{2}}{\gamma_{2}+\mu_{I}+\delta+d_{2}^{v}}} \\
& =\tau \frac{\alpha_{1}}{\alpha_{1}+\mu_{I}+\delta+d_{1}^{r}} \cdot \frac{\gamma_{2}}{\gamma_{2}+\mu_{I}+\delta+d_{2}^{v}} \cdot \frac{\left(\alpha_{1}+\mu_{I}+\delta+d_{1}^{r}\right)\left(\gamma_{2}+\mu_{I}+\delta+d_{2}^{v}\right)}{\left(\alpha_{1}+\mu_{I}+\delta+d_{1}^{r}\right)\left(\gamma_{2}+\mu_{I}+\delta+d_{2}^{v}\right)-\alpha_{1} \gamma_{2}} \\
& =\frac{\tau \alpha_{1} \gamma_{2}}{\left(\alpha_{1}+\mu_{I}+\delta+d_{1}^{r}\right)\left(\gamma_{2}+\mu_{I}+\delta+d_{2}^{v}\right)-\alpha_{1} \gamma_{2}} \text {. }
\end{aligned}
$$


This implies that the number of new infections in case (a2) is

$$
\begin{aligned}
p & \left(\frac{\mu_{E}\left(\gamma_{2}+\mu_{E}+d_{2}^{v}\right)}{\left(\alpha_{1}+\mu_{E}+d_{1}^{r}\right)\left(\gamma_{2}+\mu_{E}+d_{2}^{v}\right)-\alpha_{1} \gamma_{2}}\right. \\
& \left.\cdot \frac{\alpha_{1} \gamma_{2}}{\left(\alpha_{1}+\mu_{I}+\delta+d_{1}^{r}\right)\left(\gamma_{2}+\mu_{I}+\delta+d_{2}^{v}\right)-\alpha_{1} \gamma_{2}} \tau \beta^{T} \frac{\gamma_{2} \hat{N}_{2}^{v}}{\gamma_{2} \hat{N}_{2}^{v}+\alpha_{2} \hat{N}_{2}^{r}}\right) \\
+(1-p) & \left(\frac{\mu_{E}\left(\gamma_{2}+\mu_{E}+d_{2}^{v}\right)}{\left(\alpha_{1}+\mu_{E}+d_{1}^{r}\right)\left(\gamma_{2}+\mu_{E}+d_{2}^{v}\right)-\alpha_{1} \gamma_{2}}\right. \\
& \left.\cdot \frac{\alpha_{1} \gamma_{2}}{\left(\alpha_{1}+\mu_{A}+d_{1}^{r}\right)\left(\gamma_{2}+\mu_{A}+d_{2}^{v}\right)-\alpha_{1} \gamma_{2}} \tau \rho \beta^{T} \frac{\gamma_{2} \hat{N}_{2}^{v}}{\gamma_{2} \hat{N}_{2}^{v}+\alpha_{2} \hat{N}_{2}^{r}}\right) .
\end{aligned}
$$

Next, we go through the possible scenarios in case (B). In case (b1), the expected duration of infection of an individual in class $I_{1}^{v}$ after the $(2 n+1)$ th travel $(n=0,1, \ldots)$ is

$$
\frac{\gamma_{2}}{\gamma_{2}+\mu_{I}+\delta+d_{2}^{v}}\left(\frac{\alpha_{1}}{\alpha_{1}+\mu_{I}+\delta+d_{1}^{r}} \frac{\gamma_{2}}{\gamma_{2}+\mu_{I}+\delta+d_{2}^{v}}\right)^{n} \frac{1}{\mu_{I}+\alpha_{1}+\delta+d_{1}^{r}}
$$

thus the total expected infection time arises as

$$
\begin{aligned}
& \frac{\gamma_{2}}{\gamma_{2}+\mu_{I}+\delta+d_{2}^{v}} \sum_{n=0}^{\infty}\left(\frac{\alpha_{1}}{\alpha_{1}+\mu_{I}+\delta+d_{1}^{r}} \frac{\gamma_{2}}{\gamma_{2}+\mu_{I}+\delta+d_{2}^{v}}\right)^{n} \frac{1}{\alpha_{1}+\mu_{I}+\delta+d_{1}^{r}} \\
= & \frac{\gamma_{2}}{\left(\alpha_{1}+\mu_{I}+\delta+d_{1}^{r}\right)\left(\gamma_{2}+\mu_{I}+\delta+d_{2}^{v}\right)-\alpha_{1} \gamma_{2}},
\end{aligned}
$$

and the number of new infection in case (b1) is

$$
\begin{gathered}
p\left(\frac{\mu_{E} \alpha_{1}}{\left(\alpha_{1}+\mu_{E}+d_{1}^{r}\right)\left(\gamma_{2}+\mu_{E}+d_{2}^{v}\right)-\alpha_{1} \gamma_{2}}\right. \\
\left.\cdot \frac{\gamma_{2}}{\left(\alpha_{1}+\mu_{I}+\delta+d_{1}^{r}\right)\left(\gamma_{2}+\mu_{I}+\delta+d_{2}^{v}\right)-\alpha_{1} \gamma_{2}} \beta_{1}^{r r} \frac{\hat{N}_{1}^{r}}{\hat{N}_{1}^{r}+\hat{N}_{1}^{v}}\right) \\
+(1-p)\left(\frac{\mu_{E} \alpha_{1}}{\left(\alpha_{1}+\mu_{E}+d_{1}^{r}\right)\left(\gamma_{2}+\mu_{E}+d_{2}^{v}\right)-\alpha_{1} \gamma_{2}}\right. \\
\left.\cdot \frac{\gamma_{2}}{\left(\alpha_{1}+\mu_{A}+d_{1}^{r}\right)\left(\gamma_{2}+\mu_{A}+d_{2}^{v}\right)-\alpha_{1} \gamma_{2}} \rho \beta_{1}^{r r} \frac{\hat{N}_{1}^{r}}{\hat{N}_{1}^{r}+\hat{N}_{1}^{v}}\right) .
\end{gathered}
$$

Similarly, the probability of being (symptomatic) infected just before the $(2 n+1)$ th travel $(n=0,1, \ldots)$ in case $(\mathrm{b} 2)$ is

$$
\left(\frac{\gamma_{2}}{\gamma_{2}+\mu_{I}+\delta+d_{2}^{v}} \frac{\alpha_{1}}{\alpha_{1}+\mu_{I}+\delta+d_{1}^{r}}\right)^{n} \frac{\gamma_{2}}{\gamma_{2}+\mu_{I}+\delta+d_{2}^{v}},
$$

and, moreover, the duration of the infectious period during the $(2 n+1)$ th travel is $\tau$. Thus it follows that the total expected infection time of a symptomatic infected individual in case 
(b2) is

$$
\begin{aligned}
& \sum_{n=0}^{\infty} \tau\left(\frac{\gamma_{2}}{\gamma_{2}+\mu_{I}+\delta+d_{2}^{v}} \frac{\alpha_{1}}{\alpha_{1}+\mu_{I}+\delta+d_{1}^{r}}\right)^{n} \frac{\gamma_{2}}{\gamma_{2}+\mu_{I}+\delta+d_{2}^{v}} \\
= & \frac{\tau \gamma_{2}\left(\alpha_{1}+\mu_{I}+\delta+d_{1}^{r}\right)}{\left(\alpha_{1}+\mu_{I}+\delta+d_{1}^{r}\right)\left(\gamma_{2}+\mu_{I}+\delta+d_{2}^{v}\right)-\alpha_{1} \gamma_{2}},
\end{aligned}
$$

and we obtain the number of new infections in case (b2) as

$$
\begin{aligned}
p & \left(\frac{\mu_{E} \alpha_{1}}{\left(\alpha_{1}+\mu_{E}+d_{1}^{r}\right)\left(\gamma_{2}+\mu_{E}+d_{2}^{v}\right)-\alpha_{1} \gamma_{2}}\right. \\
& \left.\cdot \frac{\gamma_{2}\left(\alpha_{1}+\mu_{I}+\delta+d_{1}^{r}\right)}{\left(\alpha_{1}+\mu_{I}+\delta+d_{1}^{r}\right)\left(\gamma_{2}+\mu_{I}+\delta+d_{2}^{v}\right)-\alpha_{1} \gamma_{2}} \tau \beta^{T} \frac{\gamma_{2} \hat{N}_{2}^{v}}{\gamma_{2} \hat{N}_{2}^{v}+\alpha_{2} \hat{N}_{2}^{r}}\right) \\
+(1-p) & \left(\frac{\mu_{E} \alpha_{1}}{\left(\alpha_{1}+\mu_{E}+d_{1}^{r}\right)\left(\gamma_{2}+\mu_{E}+d_{2}^{v}\right)-\alpha_{1} \gamma_{2}}\right. \\
& \left.\cdot \frac{\gamma_{2}\left(\alpha_{1}+\mu_{A}+d_{1}^{r}\right)}{\left(\alpha_{1}+\mu_{A}+d_{1}^{r}\right)\left(\gamma_{2}+\mu_{A}+d_{2}^{v}\right)-\alpha_{1} \gamma_{2}} \tau \rho \beta^{T} \frac{\gamma_{2} \hat{N}_{2}^{v}}{\gamma_{2} \hat{N}_{2}^{v}+\alpha_{2} \hat{N}_{2}^{r}}\right) .
\end{aligned}
$$

We arrive at the formula for $R_{11}^{r r}$ by summing the number of new infections in the different cases:

$$
\begin{aligned}
R_{11}^{r r}= & p\left(\frac{\mu_{E}}{\left(\alpha_{1}+\mu_{E}+d_{1}^{r}\right)\left(\gamma_{2}+\mu_{E}+d_{2}^{v}\right)-\alpha_{1} \gamma_{2}}\right. \\
& \cdot \frac{\left(\gamma_{2}+\mu_{E}+d_{2}^{v}\right)\left(\gamma_{2}+\mu_{I}+\delta+d_{2}^{v}\right)+\alpha_{1} \gamma_{2}}{\left(\alpha_{1}+\mu_{I}+\delta+d_{1}^{r}\right)\left(\gamma_{2}+\mu_{I}+\delta+d_{2}^{v}\right)-\alpha_{1} \gamma_{2}} \beta_{1}^{r r} \frac{\hat{N}_{1}^{r}}{\hat{N}_{1}^{r}+\hat{N}_{1}^{v}} \\
& +\frac{\mu_{E} \alpha_{1} \gamma_{2}}{\left(\alpha_{1}+\mu_{E}+d_{1}^{r}\right)\left(\gamma_{2}+\mu_{E}+d_{2}^{v}\right)-\alpha_{1} \gamma_{2}} \\
& \left.\cdot \frac{\gamma_{2}+\mu_{E}+d_{2}^{v}+\alpha_{1}+\mu_{I}+\delta+d_{1}^{r}}{\left(\alpha_{1}+\mu_{I}+\delta+d_{1}^{r}\right)\left(\gamma_{2}+\mu_{I}+\delta+d_{2}^{v}\right)-\alpha_{1} \gamma_{2}} \tau \beta^{T} \frac{\gamma_{2} \hat{N}_{2}^{v}}{\gamma_{2} \hat{N}_{2}^{v}+\alpha_{2} \hat{N}_{2}^{r}}\right) \\
& +(1-p)\left(\frac{\mu_{E}}{\left(\alpha_{1}+\mu_{E}+d_{1}^{r}\right)\left(\gamma_{2}+\mu_{E}+d_{2}^{v}\right)-\alpha_{1} \gamma_{2}}\right. \\
& \cdot \frac{\left(\gamma_{2}+\mu_{E}+d_{2}^{v}\right)\left(\gamma_{2}+\mu_{A}+d_{2}^{v}\right)+\alpha_{1} \gamma_{2}}{\left(\alpha_{1}+\mu_{A}+d_{1}^{r}\right)\left(\gamma_{2}+\mu_{A}+d_{2}^{v}\right)-\alpha_{1} \gamma_{2}} \rho \beta_{1}^{r r} \frac{\hat{N}_{1}^{r}}{\hat{N}_{1}^{r}+\hat{N}_{1}^{v}} \\
& +\frac{\mu_{E} \alpha_{1} \gamma_{2}}{\left(\alpha_{1}+\mu_{E}+d_{1}^{r}\right)\left(\gamma_{2}+\mu_{E}+d_{2}^{v}\right)-\alpha_{1} \gamma_{2}} \\
& \left.\cdot \frac{\gamma_{2}+\mu_{E}+d_{2}^{v}+\alpha_{1}+\mu_{A}+d_{1}^{r}}{\left(\alpha_{1}+\mu_{A}+d_{1}^{r}\right)\left(\gamma_{2}+\mu_{A}+d_{2}^{v}\right)-\alpha_{1} \gamma_{2}} \tau \rho \beta^{T} \frac{\gamma_{2} \hat{N}_{2}^{v}}{\gamma_{2} \hat{N}_{2}^{v}+\alpha_{2} \hat{N}_{2}^{r}}\right) .
\end{aligned}
$$

We detail the calculation of another element $R_{12}^{r v}$, which is the number of new infections in $E_{2}^{v}$ originating from $E_{1}^{r}$. We may define the two scenarios for the exposed-to-infected transition as before, and thus the above calculated probabilities for events (A) and (B) still 
hold:

$$
\begin{aligned}
& P(A)=\frac{\mu_{E}\left(\gamma_{2}+\mu_{E}+d_{2}^{v}\right)}{\left(\alpha_{1}+\mu_{E}+d_{1}^{r}\right)\left(\gamma_{2}+\mu_{E}+d_{2}^{v}\right)-\alpha_{1} \gamma_{2}}, \\
& P(B)=\frac{\mu_{E} \alpha_{1}}{\left(\alpha_{1}+\mu_{E}+d_{1}^{r}\right)\left(\gamma_{2}+\mu_{E}+d_{2}^{v}\right)-\alpha_{1} \gamma_{2}} .
\end{aligned}
$$

Again, in each case different ways of disease transmission arise. In case (A), an infected individual can transmit the disease as follows:

(a1) after an even number of completed travels (counted since the individual became infected), the individual is a member of class $I_{1}^{r}\left(A_{1}^{r}\right)$; the individual leaves region 1 and infects during travel from region 1 to region 2 , or

(a2) as a member of class $I_{2}^{v}\left(A_{2}^{v}\right)$, i.e., the individual infects in region 2 after an odd number of completed travels (counted since the individual became infected).

Similarly, in case (B), the individual can transmit the disease in one of the following ways:

(b1) after an odd number of completed travels (counted since the individual became infected), the individual is a member of class $I_{1}^{r}\left(A_{1}^{r}\right)$; the individual leaves region 1 and infects during travel from region 1 to region 2 , or

(b2) as a member of class $I_{2}^{v}\left(A_{2}^{v}\right)$; i.e., the individual infects in region 2 after an even number of completed travels (counted since the individual became infected).

The total infection time in cases (a1), (a2), (b1), and (b2) arises with very similar calculations as for element $R_{11}^{r r}$ : that is, since the time of infection during travel is $\tau$, the total infection times of a symptomatic infected individual in cases (a1) and (b1) is

$$
\begin{aligned}
& \sum_{n=0}^{\infty} \tau\left(\frac{\alpha_{1}}{\alpha_{1}+\mu_{I}+\delta+d_{1}^{r}} \frac{\gamma_{2}}{\gamma_{2}+\mu_{I}+\delta+d_{2}^{v}}\right)^{n} \frac{\alpha_{1}}{\alpha_{1}+\mu_{I}+\delta+d_{1}^{r}} \\
= & \frac{\tau \alpha_{1}\left(\gamma_{2}+\mu_{I}+\delta+d_{2}^{v}\right)}{\left(\alpha_{1}+\mu_{I}+\delta+d_{1}^{r}\right)\left(\gamma_{2}+\mu_{I}+\delta+d_{2}^{v}\right)-\alpha_{1} \gamma_{2}}
\end{aligned}
$$

and

$$
\begin{aligned}
& \sum_{n=0}^{\infty} \tau \frac{\gamma_{2}}{\gamma_{2}+\mu_{I}+\delta+d_{2}^{v}}\left(\frac{\gamma_{2}}{\gamma_{2}+\mu_{I}+\delta+d_{2}^{v}} \frac{\alpha_{1}}{\alpha_{1}+\mu_{I}+\delta+d_{1}^{r}}\right)^{n} \frac{\alpha_{1}}{\alpha_{1}+\mu_{I}+\delta+d_{1}^{r}} \\
= & \frac{\tau \alpha_{1} \gamma_{2}}{\left(\alpha_{1}+\mu_{I}+\delta+d_{1}^{r}\right)\left(\gamma_{2}+\mu_{I}+\delta+d_{2}^{v}\right)-\alpha_{1} \gamma_{2}},
\end{aligned}
$$

and the durations of infection in cases (a2) and (b2) is

$$
\begin{aligned}
& \sum_{n=0}^{\infty}\left(\frac{\alpha_{1}}{\alpha_{1}+\mu_{I}+\delta+d_{1}^{r}} \frac{\gamma_{2}}{\gamma_{2}+\mu_{I}+\delta+d_{2}^{v}}\right)^{n} \frac{\alpha_{1}}{\alpha_{1}+\mu_{I}+\delta+d_{1}^{r}} \cdot \frac{1}{\gamma_{2}+\mu_{I}+\delta+d_{2}^{v}} \\
= & \frac{\alpha_{1}}{\left(\alpha_{1}+\mu_{I}+\delta+d_{1}^{r}\right)\left(\gamma_{2}+\mu_{I}+\delta+d_{2}^{v}\right)-\alpha_{1} \gamma_{2}}
\end{aligned}
$$

and

$$
\begin{aligned}
& \sum_{n=0}^{\infty}\left(\frac{\gamma_{2}}{\gamma_{2}+\mu_{I}+\delta+d_{2}^{v}} \frac{\alpha_{1}}{\alpha_{1}+\mu_{I}+\delta+d_{1}^{r}}\right)^{n} \frac{1}{\gamma_{2}+\mu_{I}+\delta+d_{2}^{v}} \\
= & \frac{\alpha_{1}+\mu_{I}+\delta+d_{1}^{r}}{\left(\alpha_{1}+\mu_{I}+\delta+d_{1}^{r}\right)\left(\gamma_{2}+\mu_{I}+\delta+d_{2}^{v}\right)-\alpha_{1} \gamma_{2}} .
\end{aligned}
$$


Thus, the number of new infections from cases (a1) and (b1) is determined by the formulas

$$
\begin{aligned}
p & \left(\frac{\mu_{E}\left(\gamma_{2}+\mu_{E}+d_{2}^{v}\right)}{\left(\alpha_{1}+\mu_{E}+d_{1}^{r}\right)\left(\gamma_{2}+\mu_{E}+d_{2}^{v}\right)-\alpha_{1} \gamma_{2}}\right. \\
& \left.\cdot \frac{\alpha_{1}\left(\gamma_{2}+\mu_{I}+\delta+d_{2}^{v}\right)}{\left(\alpha_{1}+\mu_{I}+\delta+d_{1}^{r}\right)\left(\gamma_{2}+\mu_{I}+\delta+d_{2}^{v}\right)-\alpha_{1} \gamma_{2}} \tau \beta^{T} \frac{\alpha_{1} \hat{N}_{1}^{r}}{\alpha_{1} \hat{N}_{1}^{r}+\gamma_{1} \hat{N}_{1}^{v}}\right) \\
+(1-p) & \left(\frac{\mu_{E}\left(\gamma_{2}+\mu_{E}+d_{2}^{v}\right)}{\left(\alpha_{1}+\mu_{E}+d_{1}^{r}\right)\left(\gamma_{2}+\mu_{E}+d_{2}^{v}\right)-\alpha_{1} \gamma_{2}}\right. \\
& \left.\cdot \frac{\alpha_{1}\left(\gamma_{2}+\mu_{A}+d_{2}^{v}\right)}{\left(\alpha_{1}+\mu_{A}+d_{1}^{r}\right)\left(\gamma_{2}+\mu_{A}+d_{2}^{v}\right)-\alpha_{1} \gamma_{2}} \tau \rho \beta^{T} \frac{\alpha_{1} \hat{N}_{1}^{r}}{\alpha_{1} \hat{N}_{1}^{r}+\gamma_{1} \hat{N}_{1}^{v}}\right)
\end{aligned}
$$

and

$$
\begin{aligned}
p & \left(\frac{\mu_{E} \alpha_{1}}{\left(\alpha_{1}+\mu_{E}+d_{1}^{r}\right)\left(\gamma_{2}+\mu_{E}+d_{2}^{v}\right)-\alpha_{1} \gamma_{2}}\right. \\
& \left.\cdot \frac{\alpha_{1} \gamma_{2}}{\left(\alpha_{1}+\mu_{I}+\delta+d_{1}^{r}\right)\left(\gamma_{2}+\mu_{I}+\delta+d_{2}^{v}\right)-\alpha_{1} \gamma_{2}} \tau \beta^{T} \frac{\alpha_{1} \hat{N}_{1}^{r}}{\gamma_{1} \hat{N}_{1}^{v}+\alpha_{1} \hat{N}_{1}^{r}}\right) \\
+(1-p) & \left(\frac{\mu_{E} \alpha_{1}}{\left(\alpha_{1}+\mu_{E}+d_{1}^{r}\right)\left(\gamma_{2}+\mu_{E}+d_{2}^{v}\right)-\alpha_{1} \gamma_{2}}\right. \\
& \left.\cdot \frac{\alpha_{1} \gamma_{2}}{\left(\alpha_{1}+\mu_{A}+d_{1}^{r}\right)\left(\gamma_{2}+\mu_{A}+d_{2}^{v}\right)-\alpha_{1} \gamma_{2}} \tau \rho \beta^{T} \frac{\alpha_{1} \hat{N}_{1}^{r}}{\gamma_{1} \hat{N}_{1}^{v}+\alpha_{1} \hat{N}_{1}^{r}}\right) .
\end{aligned}
$$

We derive the number of new infections in cases (a2) and (b2) as

$$
\begin{aligned}
p & \left(\frac{\mu_{E}\left(\gamma_{2}+\mu_{E}+d_{2}^{v}\right)}{\left(\alpha_{1}+\mu_{E}+d_{1}^{r}\right)\left(\gamma_{2}+\mu_{E}+d_{2}^{v}\right)-\alpha_{1} \gamma_{2}}\right. \\
\cdot & \left.\frac{\alpha_{1}}{\left(\alpha_{1}+\mu_{I}+\delta+d_{1}^{r}\right)\left(\gamma_{2}+\mu_{I}+\delta+d_{2}^{v}\right)-\alpha_{1} \gamma_{2}} \beta_{2}^{v v} \frac{\hat{N}_{2}^{v}}{\hat{N}_{2}^{r}+\hat{N}_{2}^{v}}\right) \\
+(1-p) & \left(\frac{\mu_{E}\left(\gamma_{2}+\mu_{E}+d_{2}^{v}\right)}{\left(\alpha_{1}+\mu_{E}+d_{1}^{r}\right)\left(\gamma_{2}+\mu_{E}+d_{2}^{v}\right)-\alpha_{1} \gamma_{2}}\right. \\
& \left.\cdot \frac{\alpha_{1}}{\left(\alpha_{1}+\mu_{A}+d_{1}^{r}\right)\left(\gamma_{2}+\mu_{A}+d_{2}^{v}\right)-\alpha_{1} \gamma_{2}} \rho \beta_{2}^{v v} \frac{\hat{N}_{2}^{v}}{\hat{N}_{2}^{r}+\hat{N}_{2}^{v}}\right) .
\end{aligned}
$$

and

$$
\begin{gathered}
p\left(\frac{\mu_{E} \alpha_{1}}{\left(\alpha_{1}+\mu_{E}+d_{1}^{r}\right)\left(\gamma_{2}+\mu_{E}+d_{2}^{v}\right)-\alpha_{1} \gamma_{2}}\right. \\
\left.\cdot \frac{\alpha_{1}+\mu_{I}+\delta+d_{1}^{r}}{\left(\alpha_{1}+\mu_{I}+\delta+d_{1}^{r}\right)\left(\gamma_{2}+\mu_{I}+\delta+d_{2}^{v}\right)-\alpha_{1} \gamma_{2}} \beta_{2}^{v v} \frac{\hat{N}_{2}^{v}}{\hat{N}_{2}^{r}+\hat{N}_{2}^{v}}\right) \\
+(1-p)\left(\frac{\mu_{E} \alpha_{1}}{\left(\alpha_{1}+\mu_{E}+d_{1}^{r}\right)\left(\gamma_{2}+\mu_{E}+d_{2}^{v}\right)-\alpha_{1} \gamma_{2}}\right. \\
\left.\cdot \frac{\alpha_{1}+\mu_{A}+d_{1}^{r}}{\left(\alpha_{1}+\mu_{A}+d_{1}^{r}\right)\left(\gamma_{2}+\mu_{A}+d_{2}^{v}\right)-\alpha_{1} \gamma_{2}} \rho \beta_{2}^{v v} \frac{\hat{N}_{2}^{v}}{\hat{N}_{2}^{r}+\hat{N}_{2}^{v}}\right) .
\end{gathered}
$$


We obtain the new infections in $E_{2}^{v}$ generated by individuals in $E_{1}^{r}$ by the formula

$$
\begin{aligned}
R_{12}^{r v}= & p\left(\frac{\mu_{E} \alpha_{1}}{\left(\alpha_{1}+\mu_{E}+d_{1}^{r}\right)\left(\gamma_{2}+\mu_{E}+d_{2}^{v}\right)-\alpha_{1} \gamma_{2}}\right. \\
& \cdot \frac{\left(\gamma_{2}+\mu_{E}+d_{2}^{v}\right)\left(\gamma_{2}+\mu_{I}+\delta+d_{2}^{v}\right)+\alpha_{1} \gamma_{2}}{\left(\alpha_{1}+\mu_{I}+\delta+d_{1}^{r}\right)\left(\gamma_{2}+\mu_{I}+\delta+d_{2}^{v}\right)-\alpha_{1} \gamma_{2}} \tau \beta^{T} \frac{\alpha_{1} \hat{N}_{1}^{r}}{\gamma_{1} \hat{N}_{1}^{v}+\alpha_{1} \hat{N}_{1}^{r}} \\
& +\frac{\mu_{E} \alpha_{1}}{\left(\alpha_{1}+\mu_{E}+d_{1}^{r}\right)\left(\gamma_{2}+\mu_{E}+d_{2}^{v}\right)-\alpha_{1} \gamma_{2}} \\
& \left.\cdot \frac{\gamma_{2}+\mu_{E}+d_{2}^{v}+\alpha_{1}+\mu_{I}+\delta+d_{1}^{r}}{\left(\alpha_{1}+\mu_{I}+\delta+d_{1}^{r}\right)\left(\gamma_{2}+\mu_{I}+\delta+d_{2}^{v}\right)-\alpha_{1} \gamma_{2}} \beta_{2}^{v v} \frac{\hat{N}_{2}^{v}}{\hat{N}_{2}^{r}+\hat{N}_{2}^{v}}\right) \\
+ & (1-p)\left(\frac{\mu_{E} \alpha_{1}}{\left(\alpha_{1}+\mu_{E}+d_{1}^{r}\right)\left(\gamma_{2}+\mu_{E}+d_{2}^{v}\right)-\alpha_{1} \gamma_{2}}\right. \\
& \quad \cdot \frac{\left(\gamma_{2}+\mu_{E}+d_{2}^{v}\right)\left(\gamma_{2}+\mu_{A}+d_{2}^{v}\right)+\alpha_{1} \gamma_{2}}{\left(\alpha_{1}+\mu_{A}+d_{1}^{r}\right)\left(\gamma_{2}+\mu_{A}+d_{2}^{v}\right)-\alpha_{1} \gamma_{2}} \tau \rho \beta^{T} \frac{\alpha_{1} \hat{N}_{1}^{r}}{\alpha_{1} \hat{N}_{1}^{r}+\gamma_{1} \hat{N}_{1}^{v}} \\
& \quad+\frac{\mu_{E} \alpha_{1}}{\left(\alpha_{1}+\mu_{E}+d_{1}^{r}\right)\left(\gamma_{2}+\mu_{E}+d_{2}^{v}\right)-\alpha_{1} \gamma_{2}} \\
& \left.\cdot \frac{\gamma_{2}+\mu_{E}+d_{2}^{v}+\alpha_{1}+\mu_{A}+d_{1}^{r}}{\left(\alpha_{1}+\mu_{A}+d_{1}^{r}\right)\left(\gamma_{2}+\mu_{A}+d_{2}^{v}\right)-\alpha_{1} \gamma_{2}} \rho \beta_{2}^{v v} \frac{\hat{N}_{2}^{v}}{\hat{N}_{2}^{r}+\hat{N}_{2}^{v}}\right) .
\end{aligned}
$$

The element $R_{r v}^{11}$ can be obtained very similarly as $R_{r r}^{11}$ (change $S_{1}^{r}$ to $S_{1}^{v}$, change $\gamma_{2} S_{2}^{v}$ to $\alpha_{2} S_{2}^{r}$, change $\beta_{1}^{r r}$ to $\beta_{1}^{r v}$ ). $R_{r r}^{12}$ arises as one writes $S_{2}^{r}$ instead of $S_{2}^{v}, \gamma_{1} S_{1}^{v}$ instead of $\alpha_{1} S_{1}^{r}$, and $\beta_{2}^{v r}$ instead of $\beta_{2}^{v v}$ in the formula of $R_{r v}^{12}$. The elements of the second column can be derived by using the elements of the first column and changing the first upper index of the transmission rates from $r$ to $v$ (i.e., $\beta_{1}^{v r}$ instead of $\beta_{1}^{r r}$, etc). For an element $R_{j, k}^{m, n}$ of the third or fourth column one may consider $R_{k, j}^{m, n}$, the corresponding element which is in the first or second column, and change index 1 to 2 and index 2 to 1 .

Now we show that $\mathcal{K}=\mathcal{N}$. The first element of the first row of $\mathcal{K}$ arises as the scalar product of the first row of $\mathcal{F}$ and the first column of $\mathcal{V}^{-1}$. From section 4 we obtain

$$
\begin{aligned}
(\mathcal{F})_{1, \cdot}= & \left(0, \rho \beta_{1}^{r r} \frac{\hat{N}_{1}^{r}}{\hat{N}_{1}^{r}+\hat{N}_{1}^{v}}, \beta_{1}^{r r} \frac{\hat{N}_{1}^{r}}{\hat{N}_{1}^{r}+\hat{N}_{1}^{v}}, 0, \rho \beta_{1}^{v r} \frac{\hat{N}_{1}^{r}}{\hat{N}_{1}^{r}+\hat{N}_{1}^{v}}, \beta_{1}^{v r} \frac{\hat{N}_{1}^{r}}{\hat{N}_{1}^{r}+\hat{N}_{1}^{v}}, 0,\right. \\
& \left.\frac{\tau \beta^{T} \rho \alpha_{2} \gamma_{2} \hat{N}_{2}^{v}}{\alpha_{2} \hat{N}_{2}^{r}+\gamma_{2} \hat{N}_{2}^{v}}, \frac{\tau \beta^{T} \alpha_{2} \gamma_{2} \hat{N}_{2}^{v}}{\alpha_{2} \hat{N}_{2}^{r}+\gamma_{2} \hat{N}_{2}^{v}}, 0, \frac{\tau \beta^{T} \rho \gamma_{2}^{2} \hat{N}_{2}^{v}}{\alpha_{2} \hat{N}_{2}^{r}+\gamma_{2} \hat{N}_{2}^{v}}, \frac{\tau \beta^{T} \gamma_{2}^{2} \hat{N}_{2}^{v}}{\alpha_{2} \hat{N}_{2}^{r}+\gamma_{2} \hat{N}_{2}^{v}}\right),
\end{aligned}
$$




$$
\begin{aligned}
\left(\mathcal{V}^{-1}\right)_{,, 1}= & \left(\frac{\gamma_{2}+\mu_{E}+d_{v}^{2}}{\left(\alpha_{1}+\mu_{E}+d_{r}^{1}\right)\left(\gamma_{2}+\mu_{E}+d_{v}^{2}\right)-\alpha_{1} \gamma_{2}},\right. \\
& \frac{(1-p) \mu_{E}}{\left(\alpha_{1}+\mu_{E}+d_{1}^{r}\right)\left(\gamma_{2}+\mu_{E}+d_{2}^{v}\right)-\alpha_{1} \gamma_{2}} \cdot \frac{\left(\gamma_{2}+\mu_{E}+d_{2}^{v}\right)\left(\gamma_{2}+\mu_{A}+d_{2}^{v}\right)+\alpha_{1} \gamma_{2}}{\left(\alpha_{1}+\mu_{A}+d_{1}^{r}\right)\left(\gamma_{2}+\mu_{A}+d_{2}^{v}\right)-\alpha_{1} \gamma_{2}}, \\
& \frac{p \mu_{E}}{\left(\alpha_{1}+\mu_{E}+d_{1}^{r}\right)\left(\gamma_{2}+\mu_{E}+d_{2}^{v}\right)-\alpha_{1} \gamma_{2}} \cdot \frac{\left(\gamma_{2}+\mu_{E}+d_{2}^{v}\right)\left(\gamma_{2}+\mu_{I}+\delta+d_{2}^{v}\right)+\alpha_{1} \gamma_{2}}{\left(\alpha_{1}+\mu_{I}+\delta+d_{1}^{r}\right)\left(\gamma_{2}+\mu_{I}+\delta+d_{2}^{v}\right)-\alpha_{1} \gamma_{2}}, \\
& 0,0,0,0,0,0, \quad \frac{\alpha_{1}}{\left(\alpha_{1}+\mu_{E}+d_{r}^{1}\right)\left(\gamma_{2}+\mu_{E}+d_{v}^{2}\right)-\alpha_{1} \gamma_{2}}, \\
& \frac{(1-p) \mu_{E} \alpha_{1}}{\left(\alpha_{1}+\mu_{E}+d_{1}^{r}\right)\left(\gamma_{2}+\mu_{E}+d_{2}^{v}\right)-\alpha_{1} \gamma_{2}} \cdot \frac{\gamma_{2}+\mu_{E}+d_{2}^{v}+\alpha_{1}+\mu_{A}+d_{1}^{r}}{\left(\alpha_{1}+\mu_{A}+d_{1}^{r}\right)\left(\gamma_{2}+\mu_{A}+d_{2}^{v}\right)-\alpha_{1} \gamma_{2}}, \\
& \left.\frac{p \mu_{E} \alpha_{1}}{\left(\alpha_{1}+\mu_{E}+d_{1}^{r}\right)\left(\gamma_{2}+\mu_{E}+d_{2}^{v}\right)-\alpha_{1} \gamma_{2}} \cdot \frac{\gamma_{2}+\mu_{E}+d_{2}^{v}+\alpha_{1}+\mu_{I}+\delta+d_{1}^{r}}{\left(\alpha_{1}+\mu_{I}+\delta+d_{1}^{r}\right)\left(\gamma_{2}+\mu_{I}+\delta+d_{2}^{v}\right)-\alpha_{1} \gamma_{2}}\right)^{T},
\end{aligned}
$$

and the product indeed equals $R_{11}^{r r}$. In order to obtain the first element of the fourth row of $\mathcal{K}$, one needs to multiply the tenth row of $\mathcal{F}$ with the first column of $\mathcal{V}^{-1}$. The tenth row of $\mathcal{F}$ arises as

$$
\begin{aligned}
(\mathcal{F})_{10, \cdot}= & \left(0, \frac{\tau \beta^{T} \rho \alpha_{1}^{2} \hat{N}_{1}^{r}}{\alpha_{1} \hat{N}_{1}^{r}+\gamma_{1} \hat{N}_{1}^{v}}, \frac{\tau \beta^{T} \alpha_{1}^{2} \hat{N}_{1}^{r}}{\alpha_{1} \hat{N}_{1}^{r}+\gamma_{1} \hat{N}_{1}^{v}}, 0, \frac{\tau \beta^{T} \rho \alpha_{1} \gamma_{1} \hat{N}_{1}^{r}}{\alpha_{1} \hat{N}_{1}^{r}+\gamma_{1} \hat{N}_{1}^{v}}, \frac{\tau \beta^{T} \alpha_{1} \gamma_{1} \hat{N}_{1}^{r}}{\alpha_{1} \hat{N}_{1}^{r}+\gamma_{1} \hat{N}_{1}^{v}},\right. \\
& \left.0, \rho \beta_{2}^{r v} \frac{\hat{N}_{2}^{v}}{\hat{N}_{2}^{r}+\hat{N}_{2}^{v}}, \beta_{2}^{r v} \frac{\hat{N}_{2}^{v}}{\hat{N}_{2}^{r}+\hat{N}_{2}^{v}}, 0, \rho \beta_{2}^{v v} \frac{\hat{N}_{2}^{v}}{\hat{N}_{2}^{r}+\hat{N}_{2}^{v}}, \beta_{2}^{v v} \frac{\hat{N}_{2}^{v}}{\hat{N}_{2}^{r}+\hat{N}_{2}^{v}}\right)
\end{aligned}
$$

the product indeed gives $R_{12}^{r v}$. The equalities of the other 14 elements of $\mathcal{K}$ and $\mathcal{N}$ arise similarly.

Acknowledgment. The authors are grateful to Adam Hulman for his help with numerical simulations. The careful reading of the manuscript and helpful comments of the referees also greatly helped to improve the paper.

\section{REFERENCES}

[1] M. E. Alexander, S. M. Moghadas, G. Röst, and J. Wu, A Delay Differential Model for Pandemic Influenza with Antiviral Treatment, Bull. Math. Biol., 70 (2008), pp. 382-397.

[2] J. Arino, Diseases in metapopulations, in Modeling and Dynamics of Infectious Diseases, Ser. Contemp. Appl. Math. 11, Higher Education Press, Beijing, China, 2009, pp. 64-122.

[3] J. Arino And P. VAn Den Driessche, A multi-city epidemic model, Math. Popul. Stud., 10 (2003), pp. 175-193.

[4] D. Balcan, H. Hu, B. Goncalves, P. Bajardi, C. Poletto, J. J. Ramasco, D. Paolotti, N. Perra, M. Tizzoni, W. Van der Broeck, V. Colizza, and A. Vespignani, Seasonal transmission potential and activity peaks of the new influenza A (H1N1): A Monte Carlo likelihood analysis based on human mobility, BMC Medicine, 7 (2009), doi:10.1186/1741-7015-7-45.

[5] O. V. Baroyan, L. A. Rvachev, U. V. Basilevsky, V. V. Ermakov, K. D. Frank, M. A. Rvachev, AND V. A. ShashKov, Computer modelling of influenza epidemics for the whole country (USSR), Adv. in Appl. Probab., 3 (1971), pp. 224-226. 
[6] S. Bonhomffer and R. R. Regoes, Emergence of drug-resistant influenza virus: Population dynamical considerations, Science, 312 (2006), pp. 389-391.

[7] F. Carrat, E. Vergu, N. M. Ferguson, M. Lemaitre, S. Cauchemez, S. Leach, and A. J. VAlleron, Time lines of infection and disease in human influenza: A review of volunteer challenge studies, Am. J. Epidemiol., 167 (2008), pp. 775-785.

[8] J. Cui, Y. TAKeuchi, AND Y. SAito, Spreading disease with transport-related infection, J. Theoret. Biol., 239 (2006), pp. 376-390.

[9] O. Diekmann and J. A. P. Heesterbeek, Mathematical Epidemiology of Infectious Diseases: Model Building, Analysis and interpretation, Wiley, Chichester, UK, 2000.

[10] O. Diekmann, J. A. P. Heesterbeek, and J. A. J. Metz, On the definition and computation of the basic reproduction ratio $R 0$ in models for infectious diseases in heterogeneous populations, J. Math. Biol., 28 (1990), pp. 365-382.

[11] O. Diekmann, J. A. P. Heesterbeek, and M. G. Robert, The construction of next-generation matrices for compartmental epidemic models, J. R. Soc. Interface, 7 (2010), pp. 873-885.

[12] European Centre for Disease Prevention and Control, Risk Assessment Guidelines for Infectious Diseases Transmitted on Aircraft, http://ecdc.europa.eu/en/publications/Publications/ 0906_TER_Risk_Assessment_Guidelines_for_Infectious _Diseases_Transmitted_on_Aircraft.pdf, 2009.

[13] M. W. Hirsch And H. Smith, Monotone dynamical systems, in Handbook of Differential Equations, Ordinary Differential Equations, 2006, Vol. 2, pp. 239-357.

[14] K. Khan, J. Arino, F. Calderon, A. Chan, M. Gardam, C. Heidebrecht, W. Hu, D. A. Janes, M. Macdonald, J. Sears, P. Raposo, and S. Wang, An Analysis of Canada's Vulnerability to Emerging Infectious Disease Threats via the Global Airline Transportation Network, Technical report, Bio. Diaspora Project, St. Michael's Hospital, Toronto, ON, Canada, 2009.

[15] K. Khan, J. Arino, W. Hu, P. Raposo, J. Sears, F. Calderon, C. Heidebrecht, M. Macdonald, J. Liauw, A. Chan, And M. Gardam, Spread of a novel influenza A (H1N1) virus via global airline transportation, N. Engl. J. Med., 361 (2009), pp. 212-214.

[16] D. H. KNIPL, Fundamental properties of differential equations with dynamically defined delayed feedback, Electron. J. Qual. Theory Differential Equations, 17 (2013), pp. 1-18.

[17] D. H. Knipl And G. Röst, Multiregional SIR model with infection during transportation, Biomath., 1 (2012), 1209255.

[18] J. Liu, J. Wu, AND Y. Zhou, Modeling disease spread via transport-related infection by a delay differential equation, Rocky Mountain J. Math., 38 (2008), pp. 1525-1540.

[19] X. Liu And Y. TAKeuchi, Spread of disease with transport-related infection and entry screening, J. Theoret. Biol., 242 (2006), pp. 517-528.

[20] I. M. Longini, Jr., M. E. Halloran, A. Nizam, and Y. Yang, Containing pandemic influenza with antiviral agents, Am. J. Epidemiol., 159 (2004), pp. 623-633.

[21] Mexican Social Security Institute, website, http://www.imss.gob.mx/english/Pages/default.aspx.

[22] Y. NAKATA, On the global stability of a delayed epidemic model with transport-related infection, Nonlinear Anal. Real World Appl., 12 (2011), pp. 3028-3034.

[23] Y. NAKata And G. Röst, Global analysis for spread of infectious diseases via transportation networks, manuscript.

[24] Public Health Agency of Canada, website, http://www.phac-aspc.gc.ca/fluwatch/index-eng.php.

[25] Sh. Ruan, W. Wang, And S. A. Levin, The effect of global travel on the spread of SARS, Math. Biosci. Eng., 3 (2006) pp. 205-218.

[26] L. A. Rvachev and I. M. Longini, Jr., A mathematical model for the global spread of influenza, Math. Biosci., 75 (1985), pp. 3-22.

[27] H. L. Sмith, An Introduction to Delay Differential Equations with Applications to the Life Sciences, Springer, New York, 2010.

[28] H. L. Smith, Monotone Dynamical Systems: An Introduction to the Theory of Competitive and Cooperative Systems, AMS, Providence, RI, 1995.

[29] Statistics Canada: Canada's national statistical agency, website, http://www.statcan.gc.ca.

[30] M. Suzuki And H. Matsunaga, Stability criteria for a class of linear differential equations with offdiagonal delays, Discrete Contin. Dyn. Syst., 24 (2009), pp. 1381-1391.

[31] Y. Takeuchi, X. Liu, And J. Cui, Global dynamics of SIS models with transport-related infection, J. 
Math. Anal. Appl., 329 (2007), pp. 1460-1471.

[32] A. R. Tuite, J. Tien, M. Eisenberg, D. J. D. Earn, J. Ma, and D. N. Fisman, Cholera epidemic in Haiti, 2010: Using a transmission model to explain spatial spread of disease and identify optimal control interventions, Ann. Internal Med., 154 (2011), pp. 593-601.

[33] A. R. Tuite, A. L. Greer, M. Whelan, A. L. Winter, B. Lee, P. Yan, J. Wu, S. Moghadas, D. Buckeridge, B. Pourbohloul, And D. N. Fisman, Estimated epidemiologic parameters and morbidity associated with pandemic H1N1 influenza, CMAJ, 182 (2010).

[34] P. VAn Den Driessche And J. WAThmough, Reproduction numbers and sub-threshold endemic equilibria for compartmental models of disease transmission, Math. Biosci., 180 (2002), pp. 29-48.

[35] C. P. van der Weijden, M. L. Stein, A. J. Jacobi, M. E. E. Kretzschmar, R. Reintues, J. E. van Steenbergen, And A. Timen, Choosing pandemic parameters for pandemic preparedness planning: A comparison of pandemic scenarios prior to and following the influenza A (H1N1) 2009 pandemic, Health Policy, Available online 27 June 2012, ISSN 0168-8510, 10.1016/j.healthpol.2012.05.007.

[36] B. Wagner, B. Coburn, And S. Blower, Calculating the potential for within-flight transmission of influenza A (H1N1), BMC Med., 7 (2009).

[37] W. Wang And X.-Q. ZhaO, An age-structured epidemic model in a patchy environment, SIAM J. Appl. Math., 65 (2005), pp. 1597-1614.

[38] World Health Organization, Severe acute respiratory syndrome (SARS): Status of the outbreak and lessons for the immediate future, Geneva, May 20, 2003.

[39] World Health Organization, Global Influenza Virological Surveillance, project website, http://www. who.int/gho/epidemic_diseases/influenza/virological_surveillance/en/index.html. 